\title{
Existence and Ulam-Hyers Stability of a Fractional-Order Coupled System in the Frame of Generalized Hilfer Derivatives
}

\author{
Abdulkafi M. Saeed ${ }^{1,+}\left(\mathbb{D}\right.$, Mohammed S. Abdo ${ }^{2, *,+} \mathbb{D}$ and Mdi Begum Jeelani ${ }^{3,+}+\mathbb{D}$ \\ 1 Department of Mathematics, College of Science, Qassim University, Buraydah 51452, Saudi Arabia; \\ abdulkafi.ahmed@qu.edu.sa \\ 2 Department of Mathematics, Hodeidah University, Al-Hudaydah, Yemen \\ 3 Department of Mathematics, Imam Mohammad Ibn Saud Islamic University, Riyadh 11564, Saudi Arabia; \\ mbshaikh@imamu.edu.sa \\ * Correspondence: msabdo@hoduniv.net.ye \\ + These authors contributed equally to this work.
}

check for

updates

Citation: Saeed, A.M.; Abdo, M.S.; Jeelani, M.B. Existence and

Ulam-Hyers Stability of a Fractional-Order Coupled System in the Frame of Generalized Hilfer

Derivatives. Mathematics 2021, 9, 2543. https://doi.org/10.3390/ math9202543

Academic Editors: Michal Fečkan, Marius-F. Danca and Christopher Goodrich

Received: 23 August 2021

Accepted: 2 October 2021

Published: 10 October 2021

Publisher's Note: MDPI stays neutral with regard to jurisdictional claims in published maps and institutional affiliations.

Copyright: (c) 2021 by the authors. Licensee MDPI, Basel, Switzerland. This article is an open access article distributed under the terms and conditions of the Creative Commons Attribution (CC BY) license (https:/ / creativecommons.org/licenses/by/ $4.0 /)$.

\begin{abstract}
In this research paper, we consider a class of a coupled system of fractional integrodifferential equations in the frame of Hilfer fractional derivatives with respect to another function. The existence and uniqueness results are obtained in weighted spaces by applying Schauder's and Banach's fixed point theorems. The results reported here are more general than those found in the literature, and some special cases are presented. Furthermore, we discuss the Ulam-Hyers stability of the solution to the proposed system. Some examples are also constructed to illustrate and validate the main results.
\end{abstract}

Keywords: $\vartheta$-Hilfer fractional derivative; fractional coupled system; existence and stability of solutions; fixed point theorem

MSC: 26A33; 34A08; 34A12; 34D20; 47H10

\section{Introduction}

Recently, the theory of fractional differential equations (FDEs) has become an active space of exploration. This is because of its accurate outcomes compared with the classical differential equations (DEs). Indeed, fractional calculus has been improving the mathematical modeling of sundry phenomena in science and engineering, for more details, refer to the monographs [1-5]. The fundamental benefit of using fractional-order derivatives (FODs) rather than integer-order derivatives (IODs) is that IODs are local in nature, whereas FODs are global in nature. Numerous physical phenomena cannot be modeled for a single DE. To overcome this challenges, these kinds of phenomena can be given the assistance of coupled systems of DEs. As of late, coupled systems of FDEs have been investigated with various methodologies, see [6-10].

The existence and uniqueness results play a significant part in the theory of FDEs. The previously mentioned region has been investigated well for classical DEs. However, for FDEs, there are many theoretical aspects that need further investigation and exploration. The existence and uniqueness results of FDEs have been very much concentrated up by using Riemann-Liouville (R-L), Caputo, and Hilfer FDs, see [11-14].

Recently, notable consideration has been given to the qualitative analysis of initial and boundary value problems for FDEs with $\psi$-Caputo and $\psi$-Hilfer FDs introduced by Almedia [15] and Sousa et al. [16], respectively, see [17-24]. By considering physical phenomena which are modeled by utilizing classical FDs, the importance of $\psi$-Hilfer FD can be discussed by redesigning and remodeling such models under $\psi$-Hilfer FD.

In this regard, the most relaxing technique for stability for functional equations was presented by Ulam [25] and Hyers [26] which is famous for Hyers-Ulam (in short H-U) 
stability. The first investigation into H-U's stability for DEs was presented by Obloza [27]. Moreover, Li and Zada in [28] provided connections between the stability of U-H and uniform exponential over Banach space. These types of stability have been very wellinvestigated for FDEs, see [29-34]. The existence and stability of solutions of the following $\vartheta$-Hilfer type FDE:

$$
\left\{\begin{array}{c}
\mathbb{D}_{a^{+}, \vartheta(\varkappa)}^{\rho_{1}, \rho_{2}} v(\varkappa)=f\left(\varkappa, v(\varkappa), \mathbb{D}_{a^{+}, \vartheta(\varkappa)}^{\rho_{1}, \rho_{2}} v(\varkappa)\right), \quad \varkappa \in(a, T], \\
0<\rho_{1}<1, \quad 0 \leq \rho_{2} \leq 1, \\
\left.\mathbb{I}_{a^{+}, \vartheta(\varkappa)}^{1-\gamma} v(\varkappa)\right|_{\varkappa=a}=v_{a}, \gamma=\rho_{1}+\rho_{2}\left(1-\rho_{1}\right)
\end{array}\right.
$$

have been investigated by Vanterler et al. [35]. Abdo and Panchal in [36] proved the existence, uniqueness and Ulam-Hyers stability of the following $\vartheta$-Hilfer type fractional integrodifferential equation:

$$
\left\{\begin{array}{c}
\mathbb{D}_{a^{+}, \vartheta(\varkappa)}^{\rho_{1}, \rho_{2}} v(\varkappa)=f(\varkappa, v(\varkappa), \chi v(\varkappa)), \quad \varkappa \in(a, T] \\
0<\rho_{1}<1, \quad 0 \leq \rho_{2} \leq 1, \\
\left.\mathbb{I}_{a^{+}, \vartheta(\varkappa)}^{1-\gamma} v(\varkappa)\right|_{\varkappa=a}=v_{a}, \gamma=\rho_{1}+\rho_{2}\left(1-\rho_{1}\right)
\end{array}\right.
$$

where $\chi v(\varkappa)=\int_{0}^{\varkappa} h(\varkappa, s, v(s)) d s, \mathbb{D}_{a^{+}, \vartheta(\varkappa)}^{\rho_{1}, \rho_{2}}$ and $\mathbb{I}_{a^{+}, \vartheta(\varkappa)}^{1-\gamma}$ represent $\vartheta$-Hilfer FD and $\vartheta$ Reimann-Liouville FI, respectively.

Motivated by the above discussion, we investigate the existence, uniqueness, and $\mathrm{H}-\mathrm{U}$ stability of the solutions of a coupled system involving $a \vartheta$-Hilfer FD of the type:

$$
\left\{\begin{array}{c}
\mathbb{D}_{a^{a^{\prime}, \vartheta(\varkappa)}}^{\rho_{1}, \rho_{2}} v(\varkappa)=f\left(\varkappa, v(\varkappa), \mathbb{I}_{a^{+}, \vartheta(\varkappa)}^{\rho_{3}} \omega(\varkappa)\right), \quad \varkappa \in \mathbb{J}:=(a, b], \\
\mathbb{D}_{a_{1}^{+}, \vartheta(\vartheta)}^{\rho_{1}(\varkappa)} \omega(\varkappa)=g\left(\varkappa, \omega(\varkappa), \mathbb{I}_{a^{+}, \vartheta(\varkappa)}^{\rho^{+}} v(\varkappa)\right), \quad \varkappa \in \mathbb{J}:=(a, b], \\
\left.\mathbb{I}_{a^{+}, \vartheta(\varkappa)}^{1-\gamma} v(\varkappa)\right|_{\varkappa=a}=v_{a},\left.\mathbb{I}_{a^{+}, \vartheta(\varkappa)}^{1-\gamma} \omega(\varkappa)\right|_{\varkappa=a}=\omega_{a},
\end{array}\right.
$$

where

(i) $0<\rho_{1}<1,0 \leq \rho_{2} \leq 1, \rho_{3}>0, \gamma=\rho_{1}+\rho_{2}\left(1-\rho_{1}\right)$, and $v_{a}, \omega_{a} \in \mathbb{R}$;

(ii) $\mathbb{D}_{a^{+}, \vartheta(\varkappa)}^{\rho_{1}, \rho_{2}}$ represents the $\vartheta$-Hilfer FD of order $\rho_{1}$ and type $\rho_{2}$.

(iii) $\mathbb{I}_{a^{+}, \vartheta(\varkappa)}^{\rho_{3}}$ and $\mathbb{I}_{a^{+}, \vartheta(\varkappa)}^{1-\gamma}$ represent the $\vartheta$-R-L fractional integrals of order $\rho_{3}$ and $1-\gamma$, respectively;

(iv) $f, g: \mathbb{J} \times \mathcal{C} \times \mathcal{C} \rightarrow \mathbb{R}$ are continuous and nonlinear functions on a Banach space $\mathcal{C}$;

(v) $\vartheta \in \mathcal{C}^{1}(\mathbb{J}, \mathbb{R})$ are an increasing function with $\vartheta^{\prime}(\varkappa) \neq 0$, for all $\varkappa \in \mathbb{J}$.

We pay attention to the topic of a novel operator with respect to another function, as it covers many fractional systems that are special cases for various values of $\vartheta$. More precisely, the existence, uniqueness, and U-H stability of solutions to the system (1) are obtained in weighted spaces by using standard fixed point theorems (Banach-type and Schauder type) along with Arzelà-Ascoli's theorem.

The content of this paper is organized as follows: Section 2 presents some required results and preliminaries about $\vartheta$-Hilfer FD. Our main results for the system (1) are addressed in Section 3. Some examples to explain the acquired results are given in Section 4. In the end, we epitomize our study in the Conclusion section.

\section{Preliminaries}

In this section, we recall the concept of advanced fractional calculus. Throughout the paper, we assume that $\mathbb{J}:=(a, b] \subset \mathbb{R},(a<b), \gamma=\rho_{1}+\rho_{2}\left(1-\rho_{1}\right), 0<\rho_{1}<1$, $0 \leq \rho_{2} \leq 1$, and $\vartheta: \mathbb{J} \rightarrow \mathbb{R}$ is an increasing linear function which satisfies $\vartheta^{\prime}(\varkappa) \neq 0$, for all $\varkappa \in \mathbb{J}$. Let

$$
\mathcal{C}=\mathcal{C}(\mathbb{J}, \mathbb{R})=\left\{\phi: \mathbb{J} \rightarrow \mathbb{R} ;\|\phi\|_{\infty}=\max _{\varkappa \in \mathbb{J}}|\phi(\varkappa)|\right\}
$$


and

$$
\mathcal{C}_{1-\gamma, \vartheta}=\mathcal{C}_{1-\gamma, \vartheta}(\mathbb{J}, \mathbb{R})=\left\{\phi: \mathbb{J} \rightarrow \mathbb{R} ; \mathbb{D}_{a^{+}, \vartheta}^{\rho_{1}, \rho_{2}} \phi \in \mathcal{C} ;\|\phi\|_{1-\gamma, \vartheta}=\left\|(\vartheta(\varkappa)-\vartheta(a))^{1-\gamma} \phi(\varkappa)\right\|_{\infty}\right\} .
$$

where $0 \leq \gamma<1$. Obviously, $\mathcal{C}$ and $\mathcal{C}_{1-\gamma, \vartheta}$ are Banach spaces under $\|\phi\|_{\infty}$ and $\|\phi\|_{1-\gamma, \vartheta}$, respectively. Hence the products $\mathcal{C} \times \mathcal{C}$ and $\mathcal{C}_{1-\gamma, \vartheta} \times \mathcal{C}_{1-\gamma, \vartheta}$ are also Banach spaces with norms

$$
\left\|\left(\phi_{1}, \phi_{2}\right)\right\|_{\infty}=\left\|\phi_{1}\right\|_{\infty}+\left\|\phi_{2}\right\|_{\infty}
$$

and

$$
\left\|\left(\phi_{1}, \phi_{2}\right)\right\|_{1-\gamma, \vartheta}=\left\|\phi_{1}\right\|_{1-\gamma, \vartheta}+\left\|\phi_{2}\right\|_{1-\gamma, \vartheta}
$$

respectively. Let $z \in \mathbb{C}$ with $\operatorname{Re}(z)>0$. Then, the gamma function $\Gamma(z)$ is defined by [37]

$$
\Gamma(z)=\int_{0}^{\infty} u^{z-1} e^{-u} d u,
$$

and let $z_{1}, z_{2} \in \mathbb{C}$ with $\operatorname{Re}\left(z_{1}\right), \operatorname{Re}\left(z_{2}\right)>0$. Then, the beta function $\mathcal{B}\left(z_{1}, z_{2}\right)$ is defined by [37]

$$
\mathcal{B}\left(z_{1}, z_{2}\right)=\int_{0}^{1} u^{z_{1}-1}(1-u)^{z_{2}-1} d u .
$$

Note that, beta function and gamma function have the following relation

$$
\mathcal{B}\left(z_{1}, z_{2}\right)=\frac{\Gamma\left(z_{1}\right) \Gamma\left(z_{2}\right)}{\Gamma\left(z_{1}+z_{2}\right)} .
$$

Definition 1 ([2]). The $\vartheta-R$-L fractional integral of order $\rho_{1}>0$ for a function $\phi(\varkappa)$ is given by

$$
\mathbb{I}_{a^{+}, \vartheta(\varkappa)}^{\rho_{1}} \phi(\varkappa)=\frac{1}{\Gamma\left(\rho_{1}\right)} \int_{a}^{\varkappa} \vartheta^{\prime}(t)(\vartheta(\varkappa)-\vartheta(t))^{\rho_{1}-1} \phi(t) d t,
$$

where $\Gamma(\cdot)$ is the gamma function defined by $(2)$.

Definition 2 ([16]). The $\vartheta$-Hilfer FD of a function $\phi(\varkappa)$ of order $\rho_{1}$ and type $\rho_{2}$ is defined by

$$
\mathbb{D}_{a^{+}, \vartheta(\varkappa)}^{\rho_{1}, \rho_{2}} \phi(\varkappa)=\mathbb{I}_{a^{+}, \vartheta(\varkappa)}^{\rho_{2}\left(1-\rho_{1}\right)}\left(\frac{1}{\vartheta^{\prime}(\varkappa)} \frac{d}{d \varkappa}\right) \mathbb{I}_{a^{+}, \vartheta(\varkappa)}^{\left(1-\rho_{2}\right)\left(1-\rho_{1}\right)} \phi(\varkappa),
$$

where $0<\rho_{1}<1,0 \leq \rho_{2} \leq 1$, and $\varkappa>a$.

Lemma $1([2,16])$. Let $\rho_{1}, \eta, \delta>0$. Then

1. $\mathbb{I}_{a^{+}, \vartheta(\varkappa)}^{\rho_{1}} \mathbb{I}_{a^{+}, \vartheta(\varkappa)}^{\eta} \phi(\varkappa)=\mathbb{I}_{a^{+}, \vartheta(\varkappa)}^{\rho_{1}+\eta} \phi(\varkappa)$.

2. $\mathbb{I}_{a^{+}, \vartheta(\varkappa)}^{\rho_{1}}(\vartheta(\varkappa)-\vartheta(a))^{\delta-1}=\frac{\Gamma(\delta)}{\Gamma\left(\rho_{1}+\delta\right)}(\vartheta(\varkappa)-\vartheta(a))^{\rho_{1}+\delta-1}$.

We note also that $\mathbb{D}_{a^{+}, \vartheta(\varkappa)}^{\rho_{1}, \rho_{2}}(\vartheta(\varkappa)-\vartheta(a))^{\gamma-1}=0$, where $\gamma=\rho_{1}+\rho_{2}\left(1-\rho_{1}\right)$.

Lemma 2 ([16]). Let $\phi \in \mathcal{C}, \rho_{1} \in(0,1)$ and $\rho_{2} \in[0,1]$, then

$$
\left(\mathbb{I}_{a^{+}, \vartheta(\varkappa)}^{\rho_{1}} \mathbb{D}_{a^{+}, \vartheta(\varkappa)}^{\rho_{1}, \rho_{2}} \phi\right)(\varkappa)=\phi(\varkappa)-\frac{(\vartheta(\varkappa)-\vartheta(a))^{\zeta-1}}{\Gamma(\gamma)} \lim _{\varkappa \rightarrow a}\left(\mathbb{I}_{a^{+}, \vartheta(\varkappa)}^{\left(1-\rho_{2}\right)\left(1-\rho_{1}\right)} \phi\right)(\varkappa),
$$

where $\phi_{\vartheta}^{[n-k]}(\varkappa)=\left(\frac{1}{\vartheta^{\prime}(\varkappa)} \frac{d}{d \varkappa}\right)^{[n-k]} \phi(\varkappa)$ and $\gamma=\rho_{1}+\rho_{2}\left(1-\rho_{1}\right)$.

Theorem 1 ([38] (Banach's Theorem)). Let $\Omega \neq \varnothing$ be a closed subset of a Banach space $\mathcal{X}$. Then any contraction mapping $\mathcal{T}: \Omega \rightarrow \Omega$ has a unique fixed point. 
Theorem 2 ([39] (Schauder's Theorem)). Let $\Omega$ be a non-empty closed and convex subset of a Banach space $\mathcal{X}$. If $\mathcal{T}: \Omega \rightarrow \Omega$ is a continuous such that $\mathcal{T}(\Omega)$ is a relatively compact subset of $\mathcal{X}$, then $\mathcal{T}$ has at least one fixed point in $\Omega$.

\section{Main Results}

In this section, we establish the existence, uniqueness, and U-H stability results for the system (1). To obtain our principle results, we consider the following assumptions:

$\left(\mathbf{H y}_{1}\right) f, g: \mathbb{J} \times \mathcal{C} \times \mathcal{C} \rightarrow \mathbb{R}$ are continuous such that for each $(\varkappa, v, \omega),\left(\varkappa, v^{*}, \omega^{*}\right) \in$ $\mathbb{J} \times \mathcal{C} \times \mathcal{C}$ there exist $\kappa_{f}, \kappa_{g}, \bar{\kappa}_{f}, \bar{\kappa}_{g}>0$ with

$$
\begin{aligned}
& \left|f(\varkappa, v, \omega)-f\left(\varkappa, v^{*}, \omega^{*}\right)\right| \leq \kappa_{f}\left|v-v^{*}\right|+\bar{\kappa}_{f}\left|\omega-\omega^{*}\right|, \\
& \left|g(\varkappa, v, \omega)-g\left(\varkappa, v^{*}, \omega^{*}\right)\right| \leq \kappa_{g}\left|v-v^{*}\right|+\bar{\kappa}_{g}\left|\omega-\omega^{*}\right| .
\end{aligned}
$$

$\left(\mathrm{Hy}_{2}\right) f, g: \mathbb{J} \times \mathcal{C} \times \mathcal{C} \rightarrow \mathbb{R}$ are completely continuous such that for each $(\varkappa, v, \omega) \in$ $\mathbb{J} \times \mathcal{C} \times \mathcal{C}$ there exist $\varphi_{f}, \varphi_{g}, \bar{\varphi}_{f}, \bar{\varphi}_{g}>0$ with

$$
\begin{aligned}
& |f(\varkappa, v, \omega)| \leq \varphi_{f}|v|+\bar{\varphi}_{f}|\omega|, \\
& |g(\varkappa, v, \omega)| \leq \varphi_{g}|v|+\bar{\varphi}_{g}|\omega| .
\end{aligned}
$$

Theorem 3. Let $0<\rho_{1}<1,0 \leq \rho_{2} \leq 1$ and $\gamma=\rho_{1}+\rho_{2}\left(1-\rho_{1}\right)$. If $(v, \omega) \in$ $\mathcal{C}_{1-\gamma, \vartheta} \times \mathcal{C}_{1-\gamma, \vartheta}$ satisfies

$$
\left\{\begin{array}{c}
\mathbb{D}_{a^{+}, \vartheta(\varkappa)}^{\rho_{1}, \rho_{2}} v(\varkappa)=h_{1}(\varkappa), \quad \varkappa \in \mathbb{J}, \\
\mathbb{D}_{a^{+}, \vartheta(\varkappa)}^{\rho_{1}, \rho_{2}} \omega(\varkappa)=h_{2}(\varkappa), \quad \varkappa \in \mathbb{J}, \\
\left.\mathbb{I}_{a^{+}, \vartheta(\varkappa)}^{1-\gamma} v(\varkappa)\right|_{\varkappa=a}=v_{a}, \\
\left.\mathbb{I}_{a^{+}, \vartheta(\varkappa)}^{1-\gamma} \omega(\varkappa)\right|_{\varkappa=a}=\omega_{a},
\end{array}\right.
$$

then

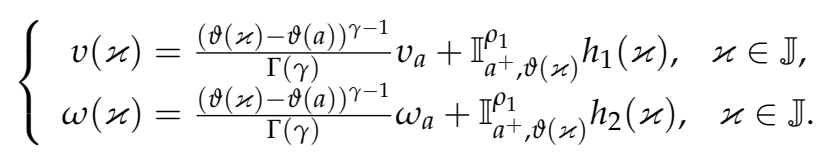

Proof. Let

$$
\left\{\begin{array}{c}
\mathbb{D}_{a^{+}, \vartheta(\varkappa)}^{\rho_{1}, \rho_{2}} v(\varkappa)=h_{1}(\varkappa), \quad \varkappa \in \mathbb{J}, \\
\left.\mathbb{I}_{a^{+}, \vartheta(\varkappa)}^{1-\gamma} v(\varkappa)\right|_{\varkappa=a}=v_{a} .
\end{array}\right.
$$

Applying the integral $\mathbb{I}_{a^{+}, \vartheta(\varkappa)}^{\rho_{1}}$ on the equation $\mathbb{D}_{a^{+}, \vartheta(\varkappa)}^{\rho_{1}, \rho_{2}} v(\varkappa)=h_{1}(\varkappa)$ and using Lemma 2, we have

$$
v(\varkappa)-\frac{(\vartheta(\varkappa)-\vartheta(a))^{\gamma-1}}{\Gamma(\gamma)} \mathbb{I}_{a^{+}, \vartheta(\varkappa)}^{\left(1-\rho_{2}\right)\left(1-\rho_{1}\right)} v(a)=\mathbb{I}_{a^{+}, \vartheta(\varkappa)}^{\rho_{1}} h_{1}(\varkappa),
$$

which implies

$$
\begin{aligned}
v(\varkappa) & =\frac{(\vartheta(\varkappa)-\vartheta(a))^{\gamma-1}}{\Gamma(\gamma)} \mathbb{I}_{a^{+}, \vartheta(\varkappa)}^{1-\gamma)} v(a)+\mathbb{I}_{a^{+}, \vartheta(\varkappa)}^{\rho_{1}} h_{1}(\varkappa) \\
& =\frac{(\vartheta(\varkappa)-\vartheta(a))^{\gamma-1}}{\Gamma(\gamma)} v_{a}+\mathbb{I}_{a^{+}, \vartheta(\varkappa)}^{\rho_{1}} h_{1}(\varkappa) .
\end{aligned}
$$

Similarly,

$$
\omega(\varkappa)=\frac{(\vartheta(\varkappa)-\vartheta(a))^{\gamma-1}}{\Gamma(\gamma)} \omega_{a}+\mathbb{I}_{a^{+}, \vartheta(\varkappa)}^{\rho_{1}} h_{2}(\varkappa)
$$




\subsection{Existence Result}

Theorem 4. Assume that $\left(H y_{1}\right)$ and $\left(H y_{2}\right)$ hold. If $\aleph_{1}:=\frac{\Lambda}{2}(\vartheta(b)-\vartheta(a))^{\rho_{1}+\rho_{3}}<1$, then system (1) has at least one solution, where $\Lambda:=\left(\left(\varphi_{f}+\varphi_{g}\right) \frac{\mathcal{B}\left(\gamma, \rho_{1}\right)}{\Gamma\left(\rho_{1}\right)}+\left(\bar{\varphi}_{f}+\bar{\varphi}_{g}\right) \frac{\mathcal{B}\left(\gamma, \rho_{1}+\rho_{3}\right)}{\Gamma\left(\rho_{1}+\rho_{3}\right)}\right)$, and $\mathcal{B}(\cdot, \cdot)$ is a beta function defined by (3).

Proof. Consider a closed ball

$$
\mathcal{S}_{\beta}=\left\{(v, \omega) \in \mathcal{C}_{1-\gamma, \vartheta} \times \mathcal{C}_{1-\gamma, \vartheta}:\|(v, \omega)\|_{\mathcal{C}_{1-\gamma, \vartheta}} \leq \beta,\|v\|_{\mathcal{C}_{1-\gamma, \vartheta}} \leq \frac{\beta}{2},\|\omega\|_{\mathcal{C}_{1-\gamma, \vartheta}} \leq \frac{\beta}{2}\right\},
$$

where $\beta \geq \frac{\aleph_{1}^{\star}}{1-\aleph_{1}}$ with $\aleph_{1}^{\star}:=\frac{\left|v_{a}\right|+\left|\omega_{a}\right|}{\Gamma(\gamma)}$. In view of Theorem 3, we transform system (1) into a fixed point system. Define the operator $\Pi=\left(\Pi_{1}, \Pi_{2}\right)$ on $\mathcal{S}_{\beta}$, where

$$
\left\{\begin{array}{l}
\Pi_{1}(v(\varkappa), \omega(\varkappa))=\frac{(\vartheta(\varkappa)-\vartheta(a))^{\gamma-1}}{\Gamma(\gamma)} v_{a}+\mathbb{I}_{a^{+}, \vartheta(\varkappa)}^{\rho_{1}} f\left(\varkappa, v(\varkappa), \mathbb{I}_{a^{+}, \vartheta(\varkappa)}^{\rho_{3}} \omega(\varkappa)\right), \quad \varkappa \in \mathbb{J}, \\
\Pi_{2}(\omega(\varkappa), v(\varkappa))=\frac{(\vartheta(\varkappa)-\vartheta(a))^{\gamma-1}}{\Gamma(\gamma)} \omega_{a}+\mathbb{I}_{a^{+}, \vartheta(\varkappa)}^{\rho_{1}} g\left(\varkappa, \omega(\varkappa), \mathbb{I}_{a^{+}, \vartheta(\varkappa)}^{\rho_{3}} v(\varkappa)\right), \quad \varkappa \in \mathbb{J} .
\end{array}\right.
$$

For any $(v, \omega) \in \mathcal{S}_{\beta}$, we have

$$
\|\Pi(v, \omega)\|_{\mathcal{C}_{1-\gamma, \vartheta}} \leq\left\|\Pi_{1}(v, \omega)\right\|_{\mathcal{C}_{1-\gamma, \vartheta}}+\left\|\Pi_{2}(\omega, v)\right\|_{\mathcal{C}_{1-\gamma, \vartheta}} .
$$

From (4), we obtain

$$
\begin{aligned}
& \left|\Pi_{1}(v(\varkappa), \omega(\varkappa))\right| \leq \frac{(\vartheta(\varkappa)-\vartheta(a))^{\gamma-1}}{\Gamma(\gamma)}\left|v_{a}\right|+\mathbb{I}_{a^{+}, \vartheta(\varkappa)}^{\rho_{1}}\left|f\left(\varkappa, v(\varkappa), \mathbb{I}_{a^{+}, \vartheta(\varkappa)}^{\rho_{3}} \omega(\varkappa)\right)\right| \\
& \leq \frac{(\vartheta(\varkappa)-\vartheta(a))^{\gamma-1}}{\Gamma(\gamma)}\left|v_{a}\right|+\mathbb{I}_{a^{+}, \vartheta(\varkappa)}^{\rho_{1}}\left(\varphi_{f}|v(\varkappa)|+\bar{\varphi}_{f} \mathbb{I}_{a^{+}, \vartheta(\varkappa)}^{\rho_{3}}|\omega(\varkappa)|\right) \\
& \leq \frac{(\vartheta(\varkappa)-\vartheta(a))^{\gamma-1}}{\Gamma(\gamma)}\left|v_{a}\right|+\varphi_{f}\left(\mathbb{I}_{a^{+}, \vartheta(\varkappa)}^{\rho_{1}}|v(\varkappa)|\right)+\bar{\varphi}_{f}\left(\mathbb{I}_{a^{+}, \vartheta(\varkappa)}^{\rho_{1}+\rho_{3}}|\omega(\varkappa)|\right) \\
& \leq \frac{(\vartheta(\varkappa)-\vartheta(a))^{\gamma-1}}{\Gamma(\gamma)}\left|v_{a}\right|+\varphi_{f}\|v\|_{\mathcal{C}_{1-\gamma, \vartheta}} \mathbb{I}_{a^{+}, \vartheta(\varkappa)}^{\rho_{1}}(\vartheta(\varkappa)-\vartheta(a))^{\gamma-1}
\end{aligned}
$$

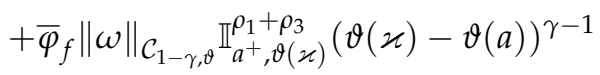

$$
\begin{aligned}
& =\frac{(\vartheta(\varkappa)-\vartheta(a))^{\gamma-1}}{\Gamma(\gamma)}\left|v_{a}\right|+\varphi_{f}\|v\|_{\mathcal{C}_{1-\gamma, \vartheta}} \frac{\Gamma(\gamma)}{\Gamma\left(\rho_{1}+\gamma\right)}(\vartheta(\varkappa)-\vartheta(a))^{\rho_{1}+\gamma-1} \\
& +\bar{\varphi}_{f}\|\omega\|_{\mathcal{C}_{1-\gamma, \vartheta}} \frac{\Gamma(\gamma)}{\Gamma\left(\rho_{1}+\rho_{3}+\gamma\right)}(\vartheta(\varkappa)-\vartheta(a))^{\rho_{1}+\rho_{3}+\gamma-1},
\end{aligned}
$$

which implies

$$
\begin{aligned}
\left\|\Pi_{1}(v, \omega)\right\|_{\mathcal{C}_{1-\gamma, \vartheta} \leq} \leq & \frac{\left|v_{a}\right|}{\Gamma(\gamma)}+\frac{\varphi_{f} \beta}{2} \frac{\Gamma(\gamma)}{\Gamma\left(\rho_{1}+\gamma\right)}(\vartheta(b)-\vartheta(a))^{\rho_{1}} \\
& +\frac{\bar{\varphi}_{f} \beta}{2} \frac{\Gamma(\gamma)}{\Gamma\left(\rho_{1}+\rho_{3}+\gamma\right)}(\vartheta(b)-\vartheta(a))^{\rho_{1}+\rho_{3}} \\
\leq & \frac{\left|v_{a}\right|}{\Gamma(\gamma)}+\frac{\beta}{2}\left(\varphi_{f} \frac{\mathcal{B}\left(\gamma, \rho_{1}\right)}{\Gamma\left(\rho_{1}\right)}+\bar{\varphi}_{f} \frac{\mathcal{B}\left(\gamma, \rho_{1}+\rho_{3}\right)}{\Gamma\left(\rho_{1}+\rho_{3}\right)}\right)(\vartheta(b)-\vartheta(a))^{\rho_{1}+\rho_{3}} .
\end{aligned}
$$

Similarly, we obtain

$$
\left\|\Pi_{2}(\omega, v)\right\|_{\mathcal{C}_{1-\gamma, \vartheta}} \leq \frac{\left|\omega_{a}\right|}{\Gamma(\gamma)}+\frac{\beta}{2}\left(\varphi_{g} \frac{\mathcal{B}\left(\gamma, \rho_{1}\right)}{\Gamma\left(\rho_{1}\right)}+\bar{\varphi}_{g} \frac{\mathcal{B}\left(\gamma, \rho_{1}+\rho_{3}\right)}{\Gamma\left(\rho_{1}+\rho_{3}\right)}\right)(\vartheta(b)-\vartheta(a))^{\rho_{1}+\rho_{3}} .
$$


In Equations (6) and (7) along with (5), give

$$
\begin{aligned}
\|\Pi(v, \omega)\|_{\mathcal{C}_{1-\gamma, \vartheta}} & \leq \frac{\left|v_{a}\right|+\left|\omega_{a}\right|}{\Gamma(\gamma)}+\frac{\beta}{2} \Lambda(\vartheta(b)-\vartheta(a))^{\rho_{1}+\rho_{3}} \\
& \leq \aleph_{1}^{\star}+\beta \aleph_{1} \leq \beta\left(1-\aleph_{1}\right)+\beta \aleph_{1}=\beta .
\end{aligned}
$$

Hence $\Pi\left(\mathcal{S}_{\beta}\right) \subset \mathcal{S}_{\beta}$.

Now, we prove that $\Pi$ is continuous and compact. Let a sequence $\left(v_{n}, \omega_{n}\right)$ in $\mathcal{S}_{\beta}$ such that $\left(v_{n}, \omega_{n}\right) \rightarrow(v, \omega)$ in $\mathcal{S}_{\beta}$ as $n \rightarrow \infty$, so, we have

$$
\begin{aligned}
& \left\|\Pi\left(v_{n}, \omega_{n}\right)(\varkappa)-\Pi(v, \omega)(\varkappa)\right\|_{\mathcal{C}_{1-\gamma, \vartheta}} \\
& =\left\|\Pi_{1}\left(v_{n}, \omega_{n}\right)(\varkappa)+\Pi_{2}\left(\omega_{n}, v_{n}\right)(\varkappa)-\Pi_{1}(v, \omega)(\varkappa)-\Pi_{2}(\omega, v)(\varkappa)\right\|_{\mathcal{C}_{1-\gamma, \vartheta}} \\
& \leq\left\|\left(\Pi_{1}\left(v_{n}, \omega_{n}\right)-\Pi_{1}(v, \omega)\right)(\varkappa)\right\|_{\mathcal{C}_{1-\gamma, \vartheta}}+\left\|\left(\Pi_{2}\left(\omega_{n}, v_{n}\right)-\Pi_{2}(\omega, v)\right)(\varkappa)\right\|_{\mathcal{C}_{1-\gamma, \vartheta}} \\
& \leq(\vartheta(\varkappa)-\vartheta(a))^{1-\gamma} \mathbb{T}_{a^{+}, \vartheta(\varkappa)}^{\rho_{1}}\left|f\left(\varkappa, v_{n}(\varkappa), \mathbb{I}_{a^{+}, \vartheta(\varkappa)}^{\rho_{3}} \omega_{n}(\varkappa)\right)-f\left(\varkappa, v(\varkappa), \mathbb{I}_{a^{+}, \vartheta(\varkappa)}^{\rho_{3}} \omega(\varkappa)\right)\right| \\
& +(\vartheta(\varkappa)-\vartheta(a))^{1-\gamma} \mathbb{T}_{a^{+}, \vartheta(\varkappa)}^{\rho_{1}}\left|g\left(\varkappa, \omega_{n}(\varkappa), \mathbb{I}_{a^{+}, \vartheta(\varkappa)}^{\rho_{3}} v_{n}(\varkappa)\right)-g\left(\varkappa, \omega(\varkappa), \mathbb{I}_{a^{+}, \vartheta(\varkappa)}^{\rho_{3}} v(\varkappa)\right)\right| \\
& \leq(\vartheta(\varkappa)-\vartheta(a))^{1-\gamma} \mathbb{I}_{a^{+}, \vartheta(\varkappa)}^{\rho_{1}}\left(\kappa_{f}\left|v_{n}(\varkappa)-v(\varkappa)\right|+\bar{\kappa}_{f} \mathbb{I}_{a^{+}, \vartheta(\varkappa)}^{\rho_{3}}\left|\omega_{n}(\varkappa)-\omega(\varkappa)\right|\right) \\
& +(\vartheta(\varkappa)-\vartheta(a))^{1-\gamma} \mathbb{I}_{a^{+}, \vartheta(\varkappa)}^{\rho_{1}}\left(\kappa_{g}\left|\omega_{n}(\varkappa)-\omega(\varkappa)\right|+\bar{\kappa}_{g} \mathbb{I}_{a^{+}, \vartheta(\varkappa)}^{\rho_{3}}\left|v_{n}(\varkappa)-v(\varkappa)\right|\right) \\
& \leq(\vartheta(\varkappa)-\vartheta(a))^{1-\gamma}\left(\kappa_{f}\left\|v_{n}-v\right\|_{\mathcal{C}_{1-\gamma, \vartheta}} \mathbb{I}_{a^{+}, \vartheta(\varkappa)}^{\rho_{1}}(\vartheta(\varkappa)-\vartheta(a))^{\gamma-1}\right. \\
& \left.+\bar{\kappa}_{f}\left\|\omega_{n}-\omega\right\|_{\mathcal{C}_{1-\gamma, \vartheta}} \mathbb{I}_{a^{+}, \vartheta(\varkappa)}^{\rho_{1}+\rho_{3}}(\vartheta(\varkappa)-\vartheta(a))^{\gamma-1}\right) \\
& +(\vartheta(\varkappa)-\vartheta(a))^{1-\gamma}\left(\kappa_{g}\left\|\omega_{n}-\omega\right\|_{\mathcal{C}_{1-\gamma, \vartheta}} \mathbb{I}_{a^{+}, \vartheta(\varkappa)}^{\rho_{1}}(\vartheta(\varkappa)-\vartheta(a))^{\gamma-1}\right. \\
& \left.+\bar{\kappa}_{g}\left\|v_{n}-v\right\|_{\mathcal{C}_{1-\gamma, \vartheta}} \mathbb{I}_{a^{+}, \vartheta(\varkappa)}^{\rho_{1}+\rho_{3}}(\vartheta(\varkappa)-\vartheta(a))^{\gamma-1}\right) \\
& \leq\left(\kappa_{f} \frac{\Gamma(\gamma)(\vartheta(b)-\vartheta(a))^{\rho_{1}}}{\Gamma\left(\rho_{1}+\gamma\right)}+\bar{\kappa}_{g} \frac{\Gamma(\gamma)(\vartheta(b)-\vartheta(a))^{\rho_{1}+\rho_{3}}}{\Gamma\left(\rho_{1}+\rho_{3}+\gamma\right)}\right)\left\|v_{n}-v\right\|_{\mathcal{C}_{1-\gamma, \vartheta}} \\
& +\left(\kappa_{g} \frac{\Gamma(\gamma)(\vartheta(b)-\vartheta(a))^{\rho_{1}}}{\Gamma\left(\rho_{1}+\gamma\right)}+\bar{\kappa}_{f} \frac{\Gamma(\gamma)(\vartheta(b)-\vartheta(a))^{\rho_{1}+\rho_{3}}}{\Gamma\left(\rho_{1}+\rho_{3}+\gamma\right)}\right)\left\|\omega_{n}-\omega\right\|_{\mathcal{C}_{1-\gamma, \vartheta}} .
\end{aligned}
$$

This implies that $\left\|\Pi\left(v_{n}, \omega_{n}\right)-\Pi(v, \omega)\right\|_{\mathcal{C}_{1-\gamma, \theta}} \rightarrow 0$ as $n \rightarrow \infty$. So, $\Pi$ is continuous. Moreover, $\Pi$ is bounded on $\mathcal{S}_{\beta}$. Therefore, $\Pi$ is uniformly bounded on $\mathcal{S}_{\beta}$.

To prove that $\Pi$ is equicontinuous, we take $\varkappa_{1}, \varkappa_{2} \in \mathbb{J}$ with $\varkappa_{1}<\varkappa_{2}$ and for any $(v, \omega) \in \mathcal{S}_{\beta}$, we obtain

$$
\begin{aligned}
& \left|\Pi(v, \omega)\left(\varkappa_{2}\right)-\Pi(v, \omega)\left(\varkappa_{1}\right)\right| \\
\leq & \left|\Pi_{1}(v, \omega)\left(\varkappa_{2}\right)-\Pi_{1}(v, \omega)\left(\varkappa_{1}\right)\right|+\left|\Pi_{2}(\omega, v)\left(\varkappa_{2}\right)-\Pi_{2}(\omega, v)\left(\varkappa_{1}\right)\right| \\
\leq & \mid \frac{\left.\left(\vartheta\left(\varkappa_{2}\right)-\vartheta(a)\right)^{\gamma-1}-\vartheta\left(\varkappa_{1}\right)-\vartheta(a)\right)^{\gamma-1}}{\Gamma(\gamma)} v_{a}+\mathbb{I}_{a^{+}, \vartheta\left(\varkappa_{2}\right)}^{\rho_{1}} f\left(\varkappa_{2}, v\left(\varkappa_{2}\right), \mathbb{I}_{a^{+}, \vartheta\left(\varkappa_{2}\right)}^{\rho_{3}} \omega\left(\varkappa_{2}\right)\right) \\
& -\mathbb{I}_{a^{+}, \vartheta\left(\varkappa_{1}\right)}^{\rho_{1}} f\left(\varkappa_{1}, v\left(\varkappa_{1}\right), \mathbb{I}_{a^{+}, \vartheta\left(\varkappa_{1}\right)}^{\rho_{3}} \omega\left(\varkappa_{1}\right)\right) \mid \\
+ & \mid \frac{\left.\left(\vartheta\left(\varkappa_{2}\right)-\vartheta(a)\right)^{\gamma-1}-\vartheta\left(\varkappa_{1}\right)-\vartheta(a)\right)^{\gamma-1}}{\Gamma(\gamma)} \omega_{a}+\mathbb{I}_{a^{+}, \vartheta\left(\varkappa_{2}\right)}^{\rho_{1}} g\left(\varkappa_{2}, \omega\left(\varkappa_{2}\right), \mathbb{I}_{a^{+}, \vartheta\left(\varkappa_{2}\right)}^{\rho_{3}} v\left(\varkappa_{2}\right)\right) \\
& -\mathbb{I}_{a^{+}, \vartheta\left(\varkappa_{1}\right)}^{\rho_{1}} g\left(\varkappa_{1}, \omega\left(\varkappa_{1}\right), \mathbb{I}_{a^{+}, \vartheta\left(\varkappa_{1}\right)}^{\rho_{3}} v\left(\varkappa_{1}\right)\right) \mid .
\end{aligned}
$$

Since $f\left(\cdot, v(\cdot), \mathbb{I}_{a^{+}, \vartheta(\cdot)}^{\rho_{3}} \omega(\cdot)\right)$ and $g\left(\cdot, \omega(\cdot), \mathbb{I}_{a^{+}, \vartheta(\cdot)}^{\rho_{3}} v(\cdot)\right)$ are continuous on $\mathbb{J}$. Therefore, there exist $\xi_{f}, \xi_{g} \in \mathbb{R}$ such that

$$
\left|f\left(\cdot, v(\cdot), \mathbb{I}_{a^{+}, \vartheta(\cdot)}^{\rho_{3}} \omega(\cdot)\right)\right| \leq \xi_{f} \text {, and }\left|g\left(\cdot, \omega(\cdot), \mathbb{I}_{a^{+}, \vartheta(\cdot)}^{\rho_{3}} v(\cdot)\right)\right| \leq \xi_{g} .
$$




\section{Hence}

$$
\begin{aligned}
& \left|\mathbb{I}_{a^{+}, \vartheta\left(\varkappa_{2}\right)}^{\rho_{1}} f\left(\varkappa_{2}, v\left(\varkappa_{2}\right), \mathbb{I}_{a^{+}, \vartheta\left(\varkappa_{2}\right)}^{\rho_{3}} \omega\left(\varkappa_{2}\right)\right)-\mathbb{I}_{a^{+}, \vartheta\left(\varkappa_{1}\right)}^{\rho_{1}} f\left(\varkappa_{1}, v\left(\varkappa_{1}\right), \mathbb{I}_{a^{+}, \vartheta\left(\varkappa_{1}\right)}^{\rho_{3}} \omega\left(\varkappa_{1}\right)\right)\right| \\
& \leq \frac{1}{\Gamma\left(\rho_{1}\right)} \int_{a}^{\varkappa_{1}} \vartheta^{\prime}(t)\left[\left(\vartheta\left(\varkappa_{1}\right)-\vartheta(t)\right)^{\rho_{1}-1}-\left(\vartheta\left(\varkappa_{2}\right)-\vartheta(t)\right)^{\rho_{1}-1}\right]\left|f\left(t, v(t), \mathbb{I}_{a^{+}, \vartheta(t)}^{\rho_{3}} \omega(t)\right)\right| d t \\
& +\frac{1}{\Gamma\left(\rho_{1}\right)} \int_{\varkappa_{1}}^{\varkappa_{2}} \vartheta^{\prime}(t)\left(\vartheta\left(\varkappa_{2}\right)-\vartheta(t)\right)^{\rho_{1}-1}\left|f\left(t, v(t), \mathbb{I}_{a^{+}, \vartheta(t)}^{\rho_{3}} \omega(t)\right)\right| d t \\
& \leq \frac{\xi_{f}}{\Gamma\left(\rho_{1}\right)} \int_{a}^{\varkappa_{1}} \vartheta^{\prime}(t)\left[\left(\vartheta\left(\varkappa_{1}\right)-\vartheta(t)\right)^{\rho_{1}-1}-\left(\vartheta\left(\varkappa_{2}\right)-\vartheta(t)\right)^{\rho_{1}-1}\right] d t \\
& +\frac{\xi_{f}}{\Gamma\left(\rho_{1}\right)} \int_{\varkappa_{1}}^{\varkappa_{2}} \vartheta^{\prime}(t)\left(\vartheta\left(\varkappa_{2}\right)-\vartheta(t)\right)^{\rho_{1}-1} d t \\
& =\frac{\xi_{f}}{\Gamma\left(\rho_{1}+1\right)}\left[\left(\vartheta\left(\varkappa_{1}\right)-\vartheta(a)\right)^{\rho_{1}}+2\left(\vartheta\left(\varkappa_{2}\right)-\vartheta\left(\varkappa_{1}\right)\right)^{\rho_{1}}-\left(\vartheta\left(\varkappa_{2}\right)-\vartheta(a)\right)^{\rho_{1}}\right] \\
& \leq \frac{2 \xi_{f}}{\Gamma\left(\rho_{1}+1\right)}\left(\vartheta\left(\varkappa_{2}\right)-\vartheta\left(\varkappa_{1}\right)\right)^{\rho_{1}} .
\end{aligned}
$$

Similarly,

$$
\begin{aligned}
& \left|\mathbb{I}_{a^{+}, \vartheta\left(\varkappa_{2}\right)}^{\rho_{1}} g\left(\varkappa_{2}, \omega\left(\varkappa_{2}\right), \mathbb{I}_{a^{+}, \vartheta\left(\varkappa_{2}\right)}^{\rho_{3}} v\left(\varkappa_{2}\right)\right)-\mathbb{I}_{a^{+}, \vartheta\left(\varkappa_{1}\right)}^{\rho_{1}} g\left(\varkappa_{1}, \omega\left(\varkappa_{1}\right), \mathbb{I}_{a^{+}, \vartheta\left(\varkappa_{1}\right)}^{\rho_{3}} v\left(\varkappa_{1}\right)\right)\right| \\
& \leq \frac{2 \xi_{g}}{\Gamma\left(\rho_{1}+1\right)}\left(\vartheta\left(\varkappa_{2}\right)-\vartheta\left(\varkappa_{1}\right)\right)^{\rho_{1}} .
\end{aligned}
$$

Substituting (10) and (11) into (9), we obtain

$$
\begin{aligned}
\left|\Pi(v, \omega)\left(\varkappa_{2}\right)-\Pi(v, \omega)\left(\varkappa_{1}\right)\right| \leq & \frac{\left(\vartheta\left(\varkappa_{2}\right)-\vartheta(a)\right)^{\gamma-1}-\left(\vartheta\left(\varkappa_{1}\right)-\vartheta(a)\right)^{\gamma-1}}{\Gamma(\gamma)}\left(v_{a}+\omega_{a}\right) \\
& +\frac{2\left(\xi_{f}+\xi_{g}\right)}{\Gamma\left(\rho_{1}+1\right)}\left(\vartheta\left(\varkappa_{2}\right)-\vartheta\left(\varkappa_{1}\right)\right)^{\rho_{1}} .
\end{aligned}
$$

Thus, $\left|\Pi(v, \omega)\left(\varkappa_{2}\right)-\Pi(v, \omega)\left(\varkappa_{1}\right)\right| \rightarrow 0$, as $\varkappa_{1} \rightarrow \varkappa_{2}$. Thus, $\Pi$ is relatively compact on $\mathcal{S}_{\beta}$. It follows that $\Pi$ is completely continuous due to the Arzela-Ascolli theorem. An application Theorem 2 shows that system (1) has at least one solution.

\subsection{Uniqueness Result}

Theorem 5. Assume that $\left(H y_{1}\right)$ holds. If $\max _{\varkappa \in \mathbb{J}}\left\{\zeta_{1}, \zeta_{2}\right\}=\zeta<1$, then the system (1) has a unique solution on $\mathbb{J}$, where

$$
\begin{aligned}
& \zeta_{1}:=\frac{(\vartheta(b)-\vartheta(a))^{\rho_{1}}}{\Gamma\left(\rho_{1}+1\right)} \kappa_{f}+\frac{(\vartheta(b)-\vartheta(a))^{\rho_{1}+\rho_{3}}}{\Gamma\left(\rho_{1}+\rho_{3}+1\right)} \bar{\kappa}_{g}, \\
& \zeta_{2}:=\frac{(\vartheta(b)-\vartheta(a))^{\rho_{1}}}{\Gamma\left(\rho_{1}+1\right)} \kappa_{g}+\frac{(\vartheta(b)-\vartheta(a))^{\rho_{1}+\rho_{3}}}{\Gamma\left(\rho_{1}+\rho_{3}+1\right)} \bar{\kappa}_{f}
\end{aligned}
$$

Proof. To demonstrate the desired result, we show that $\Pi$ is a contraction. For each $\varkappa \in \mathbb{J}$ and $(v, \omega),\left(v^{\star}, \omega^{\star}\right) \in \mathcal{S}_{\beta}$, we have 


$$
\begin{aligned}
& \left\|\Pi(v, \omega)-\Pi\left(v^{\star}, \omega^{\star}\right)\right\|_{\mathcal{C}_{1-\gamma, \vartheta}} \\
& \leq\left\|\Pi_{1}(v, \omega)-\Pi_{1}\left(v^{\star}, \omega^{\star}\right)\right\|_{\mathcal{C}_{1-\gamma, \vartheta}}+\left\|\Pi_{2}(\omega, v)-\Pi_{2}\left(\omega^{\star}, v^{\star}\right)\right\|_{\mathcal{C}_{1-\gamma, \vartheta}} \\
& \leq\left\|\mathbb{I}_{a^{+}, \vartheta(\varkappa)}^{\rho_{1}} f\left(\varkappa, v(\varkappa), \mathbb{I}_{a^{+}, \vartheta(\varkappa)}^{\rho_{3}} \omega(\varkappa)\right)-\mathbb{I}_{a^{+}, \vartheta(\varkappa)}^{\rho_{1}} f\left(\varkappa, v^{\star}(\varkappa), \mathbb{I}_{a^{+}, \vartheta(\varkappa)}^{\rho_{3}} \omega^{\star}(\varkappa)\right)\right\|_{\mathcal{C}_{1-\gamma, \vartheta}} \\
& +\left\|\mathbb{I}_{a^{+}, \vartheta(\varkappa)}^{\rho_{1}} g\left(\varkappa, \omega(\varkappa), \mathbb{I}_{a^{+}, \vartheta(\varkappa)}^{\rho_{3}} v(\varkappa)\right)-\mathbb{I}_{a^{+}, \vartheta(\varkappa)}^{\rho_{1}} g\left(\varkappa, \omega^{\star}(\varkappa), \mathbb{I}_{a^{+}, \vartheta(\varkappa)}^{\rho_{3}} v^{\star}(\varkappa)\right)\right\|_{\mathcal{C}_{1-\gamma, \vartheta}} \\
& \leq \max _{\varkappa \in \mathbb{J}}(\vartheta(\varkappa)-\vartheta(a))^{1-\gamma} \mathbb{I}_{a^{+}, \vartheta(\varkappa)}^{\rho_{1}}\left|f\left(\varkappa, v(\varkappa), \mathbb{I}_{a^{+}, \vartheta(\varkappa)}^{\rho_{3}} \omega(\varkappa)\right)-f\left(\varkappa, v^{\star}(\varkappa), \mathbb{I}_{a^{+}, \vartheta(\varkappa)}^{\rho_{3}} \omega^{\star}(\varkappa)\right)\right| \\
& +\max _{\varkappa \in \mathbb{J}}(\vartheta(\varkappa)-\vartheta(a))^{1-\gamma} \mathbb{I}_{a^{+}, \vartheta(\varkappa)}^{\rho_{1}}\left|g\left(\varkappa, \omega(\varkappa), \mathbb{I}_{a^{+}, \vartheta(\varkappa)}^{\rho_{3}} v(\varkappa)\right)-g\left(\varkappa, \omega^{\star}(\varkappa), \mathbb{I}_{a^{+}, \vartheta(\varkappa)}^{\rho_{3}} v^{\star}(\varkappa)\right)\right| \\
& \leq \max _{\varkappa \in \mathbb{J}}(\vartheta(\varkappa)-\vartheta(a))^{1-\gamma} \mathbb{I}_{a^{+}, \vartheta(\varkappa)}^{\rho_{1}}\left[\kappa_{f}\left|v(\varkappa)-v^{*}(\varkappa)\right|+\bar{\kappa}_{f} \mathbb{I}_{a^{+}, \vartheta(\varkappa)}^{\rho_{3}}\left|\omega(\varkappa)-\omega^{*}(\varkappa)\right|\right] \\
& +\max _{\varkappa \in \mathbb{J}}(\vartheta(\varkappa)-\vartheta(a))^{1-\gamma} \mathbb{I}_{a^{+}, \vartheta(\varkappa)}^{\rho_{1}}\left[\kappa_{g}\left|\omega(\varkappa)-\omega^{*}(\varkappa)\right|+\bar{\kappa}_{g} \mathbb{I}_{a^{+}, \vartheta(\varkappa)}^{\rho_{3}}\left|v(\varkappa)-v^{*}(\varkappa)\right|\right] \\
& \leq \frac{(\vartheta(b)-\vartheta(a))^{\rho_{1}}}{\Gamma\left(\rho_{1}+1\right)} \kappa_{f}\left\|v-v^{*}\right\|_{\mathcal{C}_{1-\gamma, \vartheta}}+\frac{(\vartheta(b)-\vartheta(a))^{\rho_{1}+\rho_{3}}}{\Gamma\left(\rho_{1}+\rho_{3}+1\right)} \bar{\kappa}_{f}\left\|\omega-\omega^{*}\right\|_{\mathcal{C}_{1-\gamma, \vartheta}} \\
& +\frac{(\vartheta(b)-\vartheta(a))^{\rho_{1}}}{\Gamma\left(\rho_{1}+1\right)} \kappa_{g}\left\|\omega-\omega^{*}\right\|_{\mathcal{C}_{1-\gamma, \vartheta}}+\frac{(\vartheta(b)-\vartheta(a))^{\rho_{1}+\rho_{3}}}{\Gamma\left(\rho_{1}+\rho_{3}+1\right)} \bar{\kappa}_{g}\left\|v-v^{*}\right\|_{\mathcal{C}_{1-\gamma, \vartheta}} \\
& =\zeta_{1}\left\|v-v^{*}\right\|_{\mathcal{C}_{1-\gamma, \vartheta}}+\zeta_{2}\left\|\omega-\omega^{*}\right\|_{\mathcal{C}_{1-\gamma, \vartheta^{\prime}}} \\
& \quad \text { which implies }
\end{aligned}
$$

$$
\left\|\Pi(v, \omega)-\Pi\left(v^{\star}, \omega^{\star}\right)\right\|_{\mathcal{C}_{1-\gamma, \vartheta}} \leq \zeta\left\|(v, \omega)-\left(v^{*}, \omega^{*}\right)\right\|_{\mathcal{C}_{1-\gamma, \vartheta}} .
$$

Since $\zeta<1, \Pi$ is a contraction map. Thus, a unique solution exists on $\mathbb{J}$ for system (1) in view of Theorem 1 , and this completes the proof.

\subsection{Special Cases}

In this subsection, we present some special cases according to our previous findings:

Case 1: If $\vartheta(\varkappa)=\varkappa$, then the system (1) is reduced to a Hilfer type coupled system of FIDE of the form

$$
\left\{\begin{array}{cc}
\mathbb{D}_{a^{+}, \rho_{2}}^{\rho_{1}, \rho_{2}} v(\varkappa)=f\left(\varkappa, v(\varkappa), \mathbb{I}_{a_{3}^{+}, \varkappa}^{\rho_{3}} \omega(\varkappa)\right), & \varkappa \in \mathbb{J}, \\
\mathbb{D}_{a^{+,}, \varkappa}^{\rho_{1}, \rho_{2}} \omega(\varkappa)=g\left(\varkappa, \omega(\varkappa), \mathbb{I}_{a^{+}, \varkappa}^{\rho^{+}} v(\varkappa)\right), & \varkappa \in \mathbb{J}, \\
\left.\mathbb{I}_{a^{+}, \varkappa}^{1-\gamma} v(\varkappa)\right|_{\varkappa=a}=v_{a},\left.\mathbb{I}_{a^{+}, \varkappa}^{1-\gamma} \omega(\varkappa)\right|_{\varkappa=a}=\omega_{a},
\end{array}\right.
$$

where $\mathbb{D}_{a^{+}, \varkappa}^{\rho_{1}, \rho_{2}}$ and $\mathbb{I}_{a^{+}, \varkappa}^{1-\gamma}$ represent the Hilfer FD of order $\left(\rho_{1}, \rho_{2}\right)$ and the R-L fractional integral of order $1-\gamma$, respectively (see [5]). Therefore, the results in Theorems 4 and 5 can be presented by

$$
\begin{cases}v(\varkappa) & =\frac{(\varkappa-a)^{\gamma-1}}{\Gamma(\gamma)} v_{a}+\mathbb{I}_{a^{+}, \varkappa}^{\rho_{1}} f\left(\varkappa, v(\varkappa), \mathbb{I}_{a^{+}, \varkappa}^{\rho_{3}} \omega(\varkappa)\right), \quad \varkappa \in \mathbb{J}, \\ \omega(\varkappa) & =\frac{(\varkappa-a)^{\gamma-1}}{\Gamma(\gamma)} \omega_{a}+\mathbb{I}_{a^{+}, \varkappa}^{\rho_{1}} g\left(\varkappa, \omega(\varkappa), \mathbb{I}_{a^{+}, \varkappa}^{\rho_{3}} v(\varkappa)\right), \quad \varkappa \in \mathbb{J} .\end{cases}
$$

Let

$$
\mathcal{C}_{1-\gamma}=\left\{\phi: \mathbb{J} \rightarrow \mathbb{R} ; \mathbb{D}_{a^{+}, \varkappa}^{\rho_{1}, \rho_{2}} \phi \in \mathcal{C} ;\|\phi\|_{1-\gamma}=\left\|(\varkappa-a)^{1-\gamma} \phi(\varkappa)\right\|_{\infty}\right\}, 0 \leq \gamma<1 .
$$

Then the next two corollaries are a special case of the Theorems 4 and 5. 
Corollary 1. Assume that $\left(H y_{1}\right)$ and $\left(H y_{2}\right)$ are satisfied. If $\frac{\Lambda}{2}(b-a)^{\rho_{1}+\rho_{3}}<1$, then system (12) has at least one solution $(v, \omega) \in \mathcal{C}_{1-\gamma} \times \mathcal{C}_{1-\gamma}$, where $\Lambda$ as in Theorem 4.

Corollary 2. Assume that $\left(H y_{1}\right)$ and $\left(H y_{2}\right)$ are satisfied. If $\max _{\varkappa \in \mathbb{I}}\left\{\zeta_{1}^{\star}, \zeta_{2}^{\star}\right\}=\zeta^{\star}<1$, then the system (12) has a unique solution $(v, \omega) \in \mathcal{C}_{1-\gamma} \times \mathcal{C}_{1-\gamma}$, where

$$
\begin{aligned}
& \zeta_{1}^{\star}:=\frac{(b-a)^{\rho_{1}}}{\Gamma\left(\rho_{1}+1\right)} \kappa_{f}+\frac{(b-a)^{\rho_{1}+\rho_{3}}}{\Gamma\left(\rho_{1}+\rho_{3}+1\right)} \bar{\kappa}_{g}, \\
& \zeta_{2}^{\star}:=\frac{(b-a)^{\rho_{1}}}{\Gamma\left(\rho_{1}+1\right)} \kappa_{g}+\frac{(b-a)^{\rho_{1}+\rho_{3}}}{\Gamma\left(\rho_{1}+\rho_{3}+1\right)} \bar{\kappa}_{f} .
\end{aligned}
$$

Case 2: Let $a>0$, and $\vartheta(\varkappa)=\log \varkappa$, then the system (1) is reduced to a HilferHadamard type coupled system of FIDE of the form

$$
\left\{\begin{array}{c}
\mathbb{D}_{a^{+}, \log \varkappa}^{\rho_{1}, \rho_{2}} v(\varkappa)=f\left(\varkappa, v(\varkappa), \mathbb{I}_{a^{+}, \log \varkappa}^{\rho_{3}} \omega(\varkappa)\right), \quad \varkappa \in \mathbb{J}, \\
\mathbb{D}_{a^{+}, \log \varkappa}^{\rho_{1}, \rho_{2}} \omega(\varkappa)=g\left(\varkappa, \omega(\varkappa), \mathbb{I}_{a^{+}, \log \varkappa}^{\rho_{1}} v(\varkappa)\right), \quad \varkappa \in \mathbb{J}, \\
\left.\mathbb{I}_{a^{+}, \log \varkappa}^{-1} v(\varkappa)\right|_{\varkappa=a}=v_{a},\left.\mathbb{I}_{a^{+}, \log \varkappa}^{--\gamma} \omega(\varkappa)\right|_{\varkappa=a}=\omega_{a} .
\end{array}\right.
$$

where $\mathbb{D}_{a^{+}, \log \varkappa}^{\rho_{1}, \rho_{2}}$ and $\mathbb{I}_{a^{+}, \log \varkappa}^{1-\gamma}$ represent the Hilfer-Hadamard FD of order $\left(\rho_{1}, \rho_{2}\right)$ and the Hadamard fractional integral of order $1-\gamma$, respectively, (see [40,41]). Consequently, the results in Theorems 4 and 5 can be offered by

$$
\left\{\begin{aligned}
v(\varkappa) & =\frac{\left(\log \frac{\varkappa}{a}\right)^{\gamma-1}}{\Gamma(\gamma)} v_{a}+\mathbb{I}_{a^{+}, \log \varkappa}^{\rho_{1}} f\left(\varkappa, v(\varkappa), \mathbb{I}_{a^{+}, \log \varkappa}^{\rho_{3}} \omega(\varkappa)\right), \quad \varkappa \in \mathbb{J}, \\
\omega(\varkappa) & =\frac{\left(\log \frac{\varkappa}{a} \gamma^{\gamma-1}\right.}{\Gamma(\gamma)} \omega_{a}+\mathbb{I}_{a^{+}, \log \varkappa}^{\rho_{1}} g\left(\varkappa, \omega(\varkappa), \mathbb{I}_{a^{+}, \log \varkappa}^{\rho_{3}} v(\varkappa)\right), \quad \varkappa \in \mathbb{J} .
\end{aligned}\right.
$$

Let

$\mathcal{C}_{1-\gamma, \log \varkappa}=\left\{\phi: \mathbb{J} \rightarrow \mathbb{R} ; \mathbb{D}_{a^{+}, \log \varkappa}^{\rho_{1}, \rho_{2}} \phi \in \mathcal{C} ;\|\phi\|_{1-\gamma, \log \varkappa}=\left\|\left(\log \frac{\varkappa}{a}\right)^{1-\gamma} \phi(\varkappa)\right\|_{\infty}\right\}, 0 \leq \gamma<1$.

Then the following two results are a special case of the Theorems 4 and 5 .

Corollary 3. Assume that $\left(H y_{1}\right)$ and $\left(H y_{2}\right)$ hold. If $\frac{\Lambda}{2}\left(\log \frac{b}{a}\right)^{\rho_{1}+\rho_{3}}<1$, then system (13) has at least one solution $(v, \omega) \in \mathcal{C}_{1-\gamma, \log \varkappa} \times \mathcal{C}_{1-\gamma, \log \varkappa}$, where $\Lambda$ is as in Theorem 4 .

Corollary 4. Assume that $\left(H y_{1}\right)$ and $\left(H y_{2}\right)$ are satisfied. If $\max _{\varkappa \in \mathbb{J}}\left\{\zeta_{3}^{\star}, \zeta_{4}^{\star}\right\}=\bar{\zeta}<1$, then the system (13) has a unique solution in $\mathcal{C}_{1-\gamma, \log \varkappa} \times \mathcal{C}_{1-\gamma, \log \varkappa}$ where

$$
\begin{aligned}
& \zeta_{3}^{\star}:=\frac{\left(\log \frac{b}{a}\right)^{\rho_{1}}}{\Gamma\left(\rho_{1}+1\right)} \kappa_{f}+\frac{\left(\log \frac{b}{a}\right)^{\rho_{1}+\rho_{3}}}{\Gamma\left(\rho_{1}+\rho_{3}+1\right)} \bar{\kappa}_{g}, \\
& \zeta_{4}^{\star}:=\frac{\left(\log \frac{b}{a}\right)^{\rho_{1}+\rho_{3}}}{\Gamma\left(\rho_{1}+\rho_{3}+1\right)} \bar{\kappa}_{f}+\frac{\left(\log \frac{b}{a}\right)^{\rho_{1}}}{\Gamma\left(\rho_{1}+1\right)} \kappa_{g} .
\end{aligned}
$$

Case 3: If $\vartheta(\varkappa)=\varkappa^{\rho}$, for $\rho>0$, then the system (1) is reduced to a HilferKatugumpola type coupled system of FIDE of the form

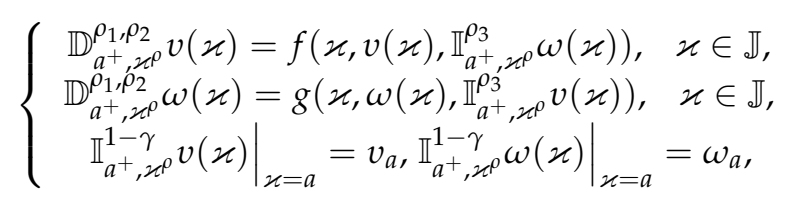


where $\mathbb{D}_{a^{+}, \varkappa^{\rho}}^{\rho_{1}, \rho_{2}}$ and $\mathbb{I}_{a^{+}, \varkappa^{\rho}}^{1-\gamma}$ represent the Hilfer-Katugumpola FD of order $\left(\rho_{1}, \rho_{2}\right)$ and the Katugumpola fractional integral of order $1-\gamma$, respectively, (see [42,43]). So, the results in Theorems 4 and 5 can be given by

$$
\begin{cases}v(\varkappa) & =\frac{\left(\varkappa^{\rho}-a^{\rho}\right)^{\gamma-1}}{\Gamma(\gamma)} v_{a}+\mathbb{I}_{a^{+}, \varkappa^{\rho}}^{\rho_{1}} f\left(\varkappa, v(\varkappa), \mathbb{I}_{a^{+}, \varkappa^{\rho}}^{\rho_{3}} \omega(\varkappa)\right), \quad \varkappa \in \mathbb{J}, \\ \omega(\varkappa) & =\frac{\left(\varkappa^{\rho}-a^{\rho}\right)^{\gamma-1}}{\Gamma(\gamma)} \omega_{a}+\mathbb{I}_{a^{+}, \varkappa^{\rho}}^{\rho^{\rho}} g\left(\varkappa, \omega(\varkappa), \mathbb{I}_{a^{+}, \varkappa^{\rho}}^{\rho^{\rho}} v(\varkappa)\right), \quad \varkappa \in \mathbb{J} .\end{cases}
$$

Let

$$
\mathcal{C}_{1-\gamma, \varkappa^{\rho}}=\left\{\phi: \mathbb{J} \rightarrow \mathbb{R} ; \mathbb{D}_{a^{+}, \varkappa^{\rho}}^{\rho_{1}, \rho_{2}} \phi \in \mathcal{C} ;\|\phi\|_{1-\gamma, \varkappa^{\rho}}=\left\|\left(\varkappa^{\rho}-a^{\rho}\right)^{1-\gamma} \phi(\varkappa)\right\|_{\infty}\right\}, 0 \leq \gamma<1 .
$$

Then the following results are a special case of the Theorems 4 and 5 .

Corollary 5. Assume that $\left(H y_{1}\right)$ and $\left(H y_{2}\right)$ hold. If $\left.\frac{\Lambda}{2}\left(b^{\rho}-a^{\rho}\right)\right)^{\rho_{1}+\rho_{3}}<1$, then system (14) has at least one solution $(v, \omega) \in \mathcal{C}_{1-\gamma, \varkappa^{\rho}} \times \mathcal{C}_{1-\gamma, \varkappa^{\rho}}$, where $\Lambda$ as in Theorem 4 .

Corollary 6. Assume that $\left(H y_{1}\right)$ and $\left(H y_{2}\right)$ are satisfied. If $\max _{\varkappa \in J}\left\{\zeta_{5}^{\star}, \zeta_{6}^{\star}\right\}=\widetilde{\zeta}<1$, then the system (14) has a unique solution in $\mathcal{C}_{1-\gamma, \varkappa^{\rho}} \times \mathcal{C}_{1-\gamma, \varkappa^{\rho}}$, where

$$
\begin{aligned}
& \zeta_{5}^{\star}:=\frac{\left(b^{\rho}-a^{\rho}\right)^{\rho_{1}}}{\Gamma\left(\rho_{1}+1\right)} \kappa_{f}+\frac{\left(b^{\rho}-a^{\rho}\right)^{\rho_{1}+\rho_{3}}}{\Gamma\left(\rho_{1}+\rho_{3}+1\right)} \bar{\kappa}_{g}, \\
& \zeta_{6}^{\star}:=\frac{\left(b^{\rho}-a^{\rho}\right)^{\rho_{1}+\rho_{3}}}{\Gamma\left(\rho_{1}+\rho_{3}+1\right)} \bar{\kappa}_{f}+\frac{\left(b^{\rho}-a^{\rho}\right)^{\rho_{1}}}{\Gamma\left(\rho_{1}+1\right)} \kappa_{g} .
\end{aligned}
$$

Remark 1. Many other special cases of function $\vartheta$ and parameter $\rho_{2}$ generate similar problems and systems some of them addressed in the literature, to name a few, the $\vartheta$-Hilfer type system (1) reduces to

(1) The $R$-L type system, for $\vartheta(\varkappa)=\varkappa$, and $\rho_{2}=0$ (see [2]);

(2) The Caputo type system, for $\vartheta(\varkappa)=\varkappa$, and $\rho_{2}=1$ (see [2]);

(3) The Hilfer type system, for $\vartheta(\varkappa)=\varkappa($ see [5]);

(4) The Katugampola type system, for $\vartheta(\varkappa)=\varkappa^{\rho}$, and $\rho_{2}=0$ (see [42]);

(5) The Caputo-Katugampola type system, for $\vartheta(\varkappa)=\varkappa^{\rho}$, and $\rho_{2}=1$ (see [44]);

(6) The Hilfer-Katugampola type system, for $\vartheta(\varkappa)=\varkappa^{\rho}$ (see [43]);

(7) The Hadamard type system, for $\vartheta(\varkappa)=\log \varkappa$, and $\rho_{2}=0$ (see [40]);

(8) The Caputo-Hadamard type system, for $\vartheta(\varkappa)=\log \varkappa$, and $\rho_{2}=1$ (see [45]);

(9) The Hilfer-Hadamard type system, for $\vartheta(\varkappa)=\log \varkappa$ (see [41]).

\subsection{U-H Stability Analysis}

In this subsection, we discuss the U-H Stability of the considered system.

Definition 3. System (1) is said to be $U$-H stable if there exists a constant $\mathrm{Y}_{1,2}=\max \left\{\mathrm{Y}_{1}, \mathrm{Y}_{2}\right\}>$ $0\left(\mathrm{Y}_{1}, \mathrm{Y}_{2}>0\right)$ such that for each $\varepsilon=\max \left\{\varepsilon_{1}, \varepsilon_{2}\right\}$, where $\varepsilon_{1}, \varepsilon_{2}>0$, and every solution $(\widetilde{v}, \widetilde{\omega}) \in$ $\mathcal{C}_{1-\gamma, \vartheta} \times \mathcal{C}_{1-\gamma, \vartheta}$ of the inequalities

$$
\left\{\begin{array}{l}
\left|\mathbb{D}_{a^{+}, \vartheta(\varkappa)}^{\rho_{1}, \rho_{2}} \widetilde{v}(\varkappa)-f\left(\varkappa, \widetilde{v}(\varkappa), \mathbb{I}_{a^{+}, \vartheta(\varkappa)}^{\rho_{3}} \widetilde{\omega}(\varkappa)\right)\right| \leq \varepsilon_{1}, \quad \varkappa \in \mathbb{J}, \\
\left|\mathbb{D}_{a^{+}, \vartheta(\varkappa)}^{\rho_{1}, \rho_{2}} \widetilde{\omega}(\varkappa)-g\left(\varkappa, \widetilde{\omega}(\varkappa), \mathbb{I}_{a^{+}, \vartheta(\varkappa)}^{\rho_{3}} \widetilde{v}(\varkappa)\right)\right| \leq \varepsilon_{2}, \quad \varkappa \in \mathbb{J},
\end{array}\right.
$$

there exists a solution $(v, \omega) \in \mathcal{C}_{1-\gamma, \vartheta} \times \mathcal{C}_{1-\gamma, \vartheta}$ of system (1) which satisfies

$$
\|(\widetilde{v}, \widetilde{\omega})-(v, \omega)\|_{\mathcal{C}_{1-\gamma, \vartheta}} \leq \mathrm{Y}_{1,2} \varepsilon
$$


Remark 2. ( $\widetilde{v}, \widetilde{\omega}) \in \mathcal{C}_{1-\gamma, \vartheta} \times \mathcal{C}_{1-\gamma, \vartheta}$ satisfies (15) if and only if there exist functions $\sigma_{1}, \sigma_{2} \in$ $\mathcal{C}_{1-\gamma, \vartheta}$ such that:

(i) $\left|\sigma_{1}(\varkappa)\right| \leq \varepsilon_{1}$, and $\left|\sigma_{2}(\varkappa)\right| \leq \varepsilon_{2}, \varkappa \in J$

(ii) For all $\varkappa \in \mathbb{J}$,

$$
\left\{\begin{array}{cc}
\mathbb{D}_{a^{+}, \vartheta(\varkappa)}^{\rho_{1}, \rho_{2}} \widetilde{v}(\varkappa)=f\left(\varkappa, \widetilde{v}(\varkappa), \mathbb{I}_{a^{+}, \vartheta(\varkappa)}^{\rho_{3}} \widetilde{\omega}(\varkappa)\right)+\sigma_{1}(\varkappa), \quad \varkappa \in \mathbb{J}, \\
\mathbb{D}_{a^{+}, \rho_{2}, \vartheta(\varkappa)}^{\rho_{1}} \widetilde{\omega}(\varkappa)=g\left(\varkappa, \widetilde{\omega}(\varkappa), \mathbb{I}_{a^{+}, \vartheta(\varkappa)}^{\rho_{3}} \widetilde{v}(\varkappa)\right)+\sigma_{2}(\varkappa), \quad \varkappa \in \mathbb{J},
\end{array}\right.
$$

Lemma 3. If $(\widetilde{v}, \widetilde{\omega}) \in \mathcal{C}_{1-\gamma, \vartheta} \times \mathcal{C}_{1-\gamma, \vartheta}$ satisfies (15), then $(\widetilde{v}, \widetilde{\omega})$ is the solution of the inequalities

$$
\left\{\begin{array}{l}
\left|\widetilde{v}(\varkappa)-\frac{(\vartheta(\varkappa)-\vartheta(a))^{\gamma-1}}{\Gamma(\gamma)} v_{a}+\mathbb{I}_{a^{+}, \vartheta(\varkappa)}^{\rho_{1}} f\left(\varkappa, \widetilde{v}(\varkappa), \mathbb{I}_{a^{+}, \vartheta(\varkappa)}^{\rho_{3}} \widetilde{\omega}(\varkappa)\right)\right| \leq \varepsilon_{1} \frac{(\vartheta(\varkappa)-\vartheta(a))^{\rho_{1}}}{\Gamma\left(\rho_{1}+1\right)}, \\
\left|\widetilde{\omega}(\varkappa)-\frac{(\vartheta(\varkappa)-\vartheta(a))^{\gamma-1}}{\Gamma(\gamma)} \omega_{a}+\mathbb{I}_{a^{+}, \vartheta(\varkappa)}^{\rho_{1}} g\left(\varkappa, \widetilde{\omega}(\varkappa), \mathbb{I}_{a^{+}, \vartheta(\varkappa)}^{\rho_{3}} \widetilde{v}(\varkappa)\right)\right| \leq \varepsilon_{2} \frac{\left(\vartheta(\varkappa)-\vartheta(a) \rho^{\rho_{1}}\right.}{\Gamma\left(\rho_{1}+1\right)} .
\end{array}\right.
$$

Proof. By virtue of Theorem 3 and Remark 2 (ii) the solution of (17) with

$$
\left.\mathbb{I}_{a^{+}, \vartheta(\varkappa)}^{1-\gamma} \widetilde{v}(\varkappa)\right|_{\varkappa=a}=v_{a},\left.\mathbb{I}_{a^{+}, \vartheta(\varkappa)}^{1-\gamma} \widetilde{\omega}(\varkappa)\right|_{\varkappa=a}=\omega_{a}
$$

is equivalent to:

$$
\left\{\begin{array}{c}
\widetilde{v}(\varkappa)=\frac{(\vartheta(\varkappa)-\vartheta(a))^{\gamma-1}}{\Gamma(\gamma)} v_{a}+\mathbb{I}_{a^{+}, \vartheta(\varkappa)}^{\rho_{1}} f\left(\varkappa, \widetilde{v}(\varkappa), \mathbb{I}_{a^{+}, \vartheta(\varkappa)}^{\rho_{3}} \widetilde{\omega}(\varkappa)\right)+\mathbb{I}_{a^{+}, \vartheta(\varkappa)}^{\rho_{1}} \sigma_{1}(\varkappa), \\
\widetilde{\omega}(\varkappa)=\frac{(\vartheta(\varkappa)-\vartheta(a))^{\gamma-1}}{\Gamma(\gamma)} \omega_{a}+\mathbb{I}_{a^{+}, \vartheta(\varkappa)}^{\rho_{1}} g\left(\varkappa, \widetilde{\omega}(\varkappa), \mathbb{I}_{a^{+}, \vartheta(\varkappa)}^{\rho_{3}} \widetilde{v}(\varkappa)\right)+\mathbb{I}_{a^{+}, \vartheta(\varkappa)}^{\rho_{1}} \sigma_{2}(\varkappa) .
\end{array}\right.
$$

Hence,

$$
\begin{aligned}
& \left|\widetilde{v}(\varkappa)-\frac{(\vartheta(\varkappa)-\vartheta(a))^{\gamma-1}}{\Gamma(\gamma)} v_{a}+\mathbb{I}_{a^{+}, \vartheta(\varkappa)}^{\rho_{1}} f\left(\varkappa, \widetilde{v}(\varkappa), \mathbb{I}_{a^{+}, \vartheta(\varkappa)}^{\rho_{3}} \widetilde{\omega}(\varkappa)\right)\right| \\
& =\left|\mathbb{I}_{a^{+}, \vartheta(\varkappa)}^{\rho_{1}} \sigma_{1}(\varkappa)\right| \\
& \leq \mathbb{I}_{a^{+}, \vartheta(\varkappa)}^{\rho_{1}}\left|\sigma_{1}(\varkappa)\right| \\
& \leq \varepsilon_{1} \frac{(\vartheta(\varkappa)-\vartheta(a))^{\rho_{1}}}{\Gamma\left(\rho_{1}+1\right)} .
\end{aligned}
$$

Similarly, we obtain

$$
\begin{aligned}
& \left|\widetilde{\omega}(\varkappa)-\frac{(\vartheta(\varkappa)-\vartheta(a))^{\gamma-1}}{\Gamma(\gamma)} \omega_{a}+\mathbb{I}_{a^{+}, \vartheta(\varkappa)}^{\rho_{1}} g\left(\varkappa, \widetilde{\omega}(\varkappa), \mathbb{I}_{a^{+}, \vartheta(\varkappa)}^{\rho_{3}} \widetilde{v}(\varkappa)\right)\right| \\
& \leq \varepsilon_{2} \frac{(\vartheta(\varkappa)-\vartheta(a))^{\rho_{1}}}{\Gamma\left(\rho_{1}+1\right)} .
\end{aligned}
$$

Theorem 6. Under the hypothesis $\left(H y_{1}\right)$, if $\left(1-\mathcal{L}_{f}\right)\left(1-\mathcal{L}_{g}\right)-\mathcal{K}_{f} \mathcal{K}_{g} \neq 0$, then the solution of the coupled system (1) is $\mathrm{H}-\mathrm{U}$ stable, where

$$
\begin{aligned}
\mathcal{L}_{f}: & =\kappa_{f} \frac{\mathcal{B}\left(\rho_{1}, \gamma\right)}{\Gamma\left(\rho_{1}\right)}(\vartheta(b)-\vartheta(a))^{\rho_{1}}, \mathcal{K}_{f}:=\bar{\kappa}_{f} \frac{\mathcal{B}\left(\rho_{1}+\rho_{3}, \gamma\right)}{\Gamma\left(\rho_{1}+\rho_{3}\right)}(\vartheta(b)-\vartheta(a))^{\rho_{1}+\rho_{3},} \\
\mathcal{L}_{g}: & =\kappa_{g} \frac{\mathcal{B}\left(\rho_{1}, \gamma\right)}{\Gamma\left(\rho_{1}\right)}(\vartheta(b)-\vartheta(a))^{\rho_{1}}, \mathcal{K}_{g}:=\bar{\kappa}_{g} \frac{\mathcal{B}\left(\rho_{1}+\rho_{3}, \gamma\right)}{\Gamma\left(\rho_{1}+\rho_{3}\right)}(\vartheta(b)-\vartheta(a))^{\rho_{1}+\rho_{3}} .
\end{aligned}
$$


Proof. Let $(\widetilde{v}, \widetilde{\omega}) \in \mathcal{C}_{1-\gamma, \vartheta} \times \mathcal{C}_{1-\gamma, \vartheta}$ satisfies (15), and let $(v, \omega) \in \mathcal{C}_{1-\gamma, \vartheta} \times \mathcal{C}_{1-\gamma, \vartheta}$ the unique solution of the system

$$
\left\{\begin{array}{c}
\mathbb{D}_{a^{+}, \vartheta(\varkappa)}^{\rho_{1}, \rho_{2}} v(\varkappa)=f\left(\varkappa, v(\varkappa), \mathbb{I}_{a^{+}, \vartheta(\varkappa)}^{\rho_{3}} \omega(\varkappa)\right), \quad \varkappa \in \mathbb{J}, \\
\mathbb{D}_{a^{+}, \vartheta(\varkappa)}^{\rho_{1, \rho}} \omega(\varkappa)=g\left(\varkappa, \omega(\varkappa), \mathbb{I}_{a^{+}, \vartheta(\varkappa)}^{\rho_{3}} v(\varkappa)\right), \quad \varkappa \in \mathbb{J}, \\
\left.\mathbb{I}_{a^{+}, \vartheta(\varkappa)}^{1-\gamma} v(\varkappa)\right|_{\varkappa=a}=\left.\mathbb{I}_{a^{+}, \vartheta(\varkappa)}^{1-\gamma} \widetilde{v}(\varkappa)\right|_{\varkappa=a}=v_{a}, \\
\left.\mathbb{I}_{a^{+}, \vartheta(\varkappa)}^{1-\gamma} \omega(\varkappa)\right|_{\varkappa=a}=\left.\mathbb{I}_{a^{+}, \vartheta(\varkappa)}^{1-\gamma} \widetilde{\omega}(\varkappa)\right|_{\varkappa=a}=\omega_{a},
\end{array}\right.
$$

By virtue of Theorem 3, we obtain

$$
\left\{\begin{array}{c}
v(\varkappa)=\mathcal{X}_{v}+\mathbb{I}_{a^{+}, \vartheta(\varkappa)}^{\rho_{1}} f\left(\varkappa, v(\varkappa), \mathbb{I}_{a^{+}, \vartheta(\varkappa)}^{\rho_{3}} \omega(\varkappa)\right), \\
\omega(\varkappa)=\mathcal{X}_{\omega}+\mathbb{I}_{a^{+}, \vartheta(\varkappa)}^{\rho^{\prime}} g\left(\varkappa, \omega(\varkappa), \mathbb{I}_{a^{+}, \vartheta(\varkappa)}^{\rho^{+}} v(\varkappa)\right),
\end{array}\right.
$$

where

$$
\begin{gathered}
\mathcal{X}_{v}=\frac{(\vartheta(\varkappa)-\vartheta(a))^{\gamma-1}}{\Gamma(\gamma)} v_{a} \text {, and } \mathcal{X}_{\omega}=\frac{(\vartheta(\varkappa)-\vartheta(a))^{\gamma-1}}{\Gamma(\gamma)} \omega_{a} . \\
\text { If }\left.\mathbb{I}_{a^{+}, \vartheta(\varkappa)}^{1-\gamma} v(\varkappa)\right|_{\varkappa=a}=\left.\mathbb{I}_{a^{+}, \vartheta(\varkappa)}^{1-\gamma} \widetilde{v}(\varkappa)\right|_{\varkappa=a} \text { and }\left.\mathbb{I}_{a^{+}, \vartheta(\varkappa)}^{1-\gamma} \omega(\varkappa)\right|_{\varkappa=a}=\left.\mathbb{I}_{a^{+}, \vartheta(\varkappa)}^{1-\gamma} \widetilde{\omega}(\varkappa)\right|_{\varkappa=a^{\prime}}
\end{gathered}
$$
then $\mathcal{X}_{v}=\mathcal{X}_{\widetilde{v}}$ and $\mathcal{X}_{\omega}=\mathcal{X}_{\widetilde{\omega}}$. Consequently, we have

$$
\left\{\begin{array}{c}
v(\varkappa)=\mathcal{X}_{\widetilde{v}}+\mathbb{I}_{a^{+}, \vartheta(\varkappa)}^{\rho_{1}} f\left(\varkappa, v(\varkappa), \mathbb{I}_{a^{+}, \vartheta(\varkappa)}^{\rho_{3}} \omega(\varkappa)\right), \\
\omega(\varkappa)=\mathcal{X}_{\widetilde{\omega}}+\mathbb{I}_{a^{+}, \vartheta(\varkappa)}^{\rho^{+}} g\left(\varkappa, \omega(\varkappa), \mathbb{I}_{a^{+}, \vartheta(\varkappa)}^{\rho^{+}} v(\varkappa)\right),
\end{array}\right.
$$

Therefore, by (22), Lemma 3 and $\left(\mathrm{Hy}_{1}\right)$, we obtain

$$
\begin{aligned}
|\widetilde{v}(\varkappa)-v(\varkappa)| \leq & \left|\widetilde{v}(\varkappa)-\mathcal{X}_{\widetilde{v}}+\mathbb{I}_{a^{+}, \vartheta(\varkappa)}^{\rho_{1}} f\left(\varkappa, \widetilde{v}(\varkappa), \mathbb{I}_{a^{+}, \vartheta(\varkappa)}^{\rho_{3}} \widetilde{\omega}(\varkappa)\right)\right| \\
& +\mathbb{I}_{a^{+}, \vartheta(\varkappa)}^{\rho_{1}}\left|f\left(\varkappa, \widetilde{v}(\varkappa), \mathbb{I}_{a^{+}, \vartheta(\varkappa)}^{\rho_{3}} \widetilde{\omega}(\varkappa)\right)-f\left(\varkappa, v(\varkappa), \mathbb{I}_{a^{+}, \vartheta(\varkappa)}^{\rho_{3}} \omega(\varkappa)\right)\right| \\
\leq & \varepsilon_{1} \frac{(\vartheta(\varkappa)-\vartheta(a))^{\rho_{1}}}{\Gamma\left(\rho_{1}+1\right)}+\mathbb{I}_{a^{+}, \vartheta(\varkappa)}^{\rho_{1}}\left[\kappa_{f}|\widetilde{v}(\varkappa)-v(\varkappa)|+\bar{\kappa}_{f} \mathbb{I}_{a^{+}, \vartheta(\varkappa)}^{\rho_{3}}|\widetilde{\omega}(\varkappa)-\omega(\varkappa)|\right] \\
\leq & \varepsilon_{1} \frac{(\vartheta(\varkappa)-\vartheta(a))^{\rho_{1}}}{\Gamma\left(\rho_{1}+1\right)}+\kappa_{f}\|\widetilde{v}-v\|_{\mathcal{C}_{1-\gamma, \vartheta}} \mathbb{I}_{a^{+}, \vartheta(\varkappa)}^{\rho_{1}}(\vartheta(\varkappa)-\vartheta(a))^{\gamma-1} \\
& +\bar{\kappa}_{f}\|\widetilde{\omega}-\omega\|_{\mathcal{C}_{1-\gamma, \vartheta}} \mathbb{I}_{a^{+}, \vartheta(\varkappa)}^{\rho_{1}+\rho_{3}}(\vartheta(\varkappa)-\vartheta(a))^{\gamma-1} \\
= & \varepsilon_{1} \frac{(\vartheta(\varkappa)-\vartheta(a))^{\rho_{1}}}{\Gamma\left(\rho_{1}+1\right)}+\kappa_{f}\|\widetilde{v}-v\|_{\mathcal{C}_{1-\gamma, \vartheta}} \frac{\mathcal{B}\left(\rho_{1}, \gamma\right)}{\Gamma\left(\rho_{1}\right)}(\vartheta(\varkappa)-\vartheta(a))^{\rho_{1}+\gamma-1} \\
& +\bar{\kappa}_{f}\|\widetilde{\omega}-\omega\|_{\mathcal{C}_{1-\gamma, \vartheta}} \frac{\mathcal{B}\left(\rho_{1}+\rho_{3}, \gamma\right)}{\Gamma\left(\rho_{1}+\rho_{3}\right)}(\vartheta(\varkappa)-\vartheta(a))^{\rho_{1}+\rho_{3}+\gamma-1 .} .
\end{aligned}
$$

Thus

$$
\begin{aligned}
\|\widetilde{v}-v\|_{\mathcal{C}_{1-\gamma, \vartheta}} \leq & \varepsilon_{1} \frac{(\vartheta(b)-\vartheta(a))^{\rho_{1}-\gamma+1}}{\Gamma\left(\rho_{1}+1\right)}+\kappa_{f}\|\widetilde{v}-v\|_{\mathcal{C}_{1-\gamma, \vartheta}} \frac{\mathcal{B}\left(\rho_{1}, \gamma\right)}{\Gamma\left(\rho_{1}\right)}(\vartheta(b)-\vartheta(a))^{\rho_{1}} \\
& +\bar{\kappa}_{f}\|\widetilde{\omega}-\omega\|_{\mathcal{C}_{1-\gamma, \vartheta}} \frac{\mathcal{B}\left(\rho_{1}+\rho_{3}, \gamma\right)}{\Gamma\left(\rho_{1}+\rho_{3}\right)}(\vartheta(b)-\vartheta(a))^{\rho_{1}+\rho_{3}},
\end{aligned}
$$

which implies

$$
\left(1-\mathcal{L}_{f}\right)\|\widetilde{v}-v\|_{\mathcal{C}_{1-\gamma, \vartheta}} \leq \mathrm{Y}_{1} \varepsilon_{1}+\mathcal{K}_{f}\|\widetilde{\omega}-\omega\|_{\mathcal{C}_{1-\gamma, \vartheta}}
$$

Similarly

$$
\left(1-\mathcal{L}_{g}\right)\|\widetilde{\omega}-\omega\|_{\mathcal{C}_{1-\gamma, \vartheta}} \leq \mathrm{Y}_{2} \varepsilon_{2}+\mathcal{K}_{g}\|\widetilde{v}-v\|_{\mathcal{C}_{1-\gamma, \vartheta^{\prime}}}
$$


where

$$
\mathrm{Y}_{1}=\mathrm{Y}_{2}:=\frac{(\vartheta(b)-\vartheta(a))^{\rho_{1}-\gamma+1}}{\Gamma\left(\rho_{1}+1\right)}
$$

Now, we can express (23) and (24) by

$$
\begin{aligned}
& \left(1-\mathcal{L}_{f}\right)\|\widetilde{v}-v\|_{\mathcal{C}_{1-\gamma, \vartheta}}-\mathcal{K}_{f}\|\widetilde{\omega}-\omega\|_{\mathcal{C}_{1-\gamma, \vartheta}} \leq \mathrm{Y}_{1} \varepsilon_{1} \\
& -\mathcal{K}_{g}\|\widetilde{v}-v\|_{\mathcal{C}_{1-\gamma, \vartheta}}+\left(1-\mathcal{L}_{g}\right)\|\widetilde{\omega}-\omega\|_{\mathcal{C}_{1-\gamma, \vartheta}} \leq \mathrm{Y}_{2} \varepsilon_{2} .
\end{aligned}
$$

The matrix formula of (25) and (26) is

$$
\left(\begin{array}{cc}
1-\mathcal{L}_{f} & -\mathcal{K}_{f} \\
-\mathcal{K}_{g} & 1-\mathcal{L}_{g}
\end{array}\right)\left(\begin{array}{c}
\|\widetilde{v}-v\|_{\mathcal{C}_{1-\gamma, \vartheta}} \\
\|\widetilde{\omega}-\omega\|_{\mathcal{C}_{1-\gamma, \vartheta}}
\end{array}\right) \leq\left(\begin{array}{c}
\mathrm{Y}_{1} \varepsilon_{1} \\
\mathrm{Y}_{2} \varepsilon_{2}
\end{array}\right) .
$$

It follows that

$$
\left(\begin{array}{c}
\|\widetilde{v}-v\|_{\mathcal{C}_{1-\gamma, \vartheta}} \\
\|\widetilde{\omega}-\omega\|_{\mathcal{C}_{1-\gamma, \vartheta}}
\end{array}\right) \leq \frac{1}{\Delta}\left(\begin{array}{cc}
1-\mathcal{L}_{g} & \mathcal{K}_{f} \\
\mathcal{K}_{g} & 1-\mathcal{L}_{f}
\end{array}\right)\left(\begin{array}{c}
\mathrm{Y}_{1} \varepsilon_{1} \\
\mathrm{Y}_{2} \varepsilon_{2}
\end{array}\right),
$$

where $\Delta=\left(1-\mathcal{L}_{f}\right)\left(1-\mathcal{L}_{g}\right)-\mathcal{K}_{f} \mathcal{K}_{g} \neq 0$. Hence

$$
\|\widetilde{v}-v\|_{\mathcal{C}_{1-\gamma, \vartheta}} \leq \frac{\left(1-\mathcal{L}_{g}\right) Y_{1} \varepsilon_{1}}{\Delta}+\frac{\mathcal{K}_{f} \mathrm{Y}_{2} \varepsilon_{2}}{\Delta},
$$

and

$$
\|\widetilde{\omega}-\omega\|_{\mathcal{C}_{1-\gamma, \vartheta}} \leq \frac{\mathcal{K}_{g} Y_{1} \varepsilon_{1}}{\Delta}+\frac{\left(1-\mathcal{L}_{f}\right) \mathrm{Y}_{2} \varepsilon_{2}}{\Delta},
$$

By (27) and (28), we find that

$$
\begin{aligned}
\|(\widetilde{v}, \widetilde{\omega})-(v, \omega)\|_{\mathcal{C}_{1-\gamma, \vartheta} \leq} \leq & \|\widetilde{v}-v\|_{\mathcal{C}_{1-\gamma, \vartheta}}+\|\widetilde{\omega}-\omega\|_{\mathcal{C}_{1-\gamma, \vartheta}} \\
\leq & \frac{\left(1-\mathcal{L}_{g}\right) \mathrm{Y}_{1} \varepsilon_{1}}{\Delta}+\frac{\mathcal{K}_{f} \mathrm{Y}_{2} \varepsilon_{2}}{\Delta} \\
& +\frac{\mathcal{K}_{g} \mathrm{Y}_{1} \varepsilon_{1}}{\Delta}+\frac{\left(1-\mathcal{L}_{f}\right) \mathrm{Y}_{2} \varepsilon_{2}}{\Delta} \\
\leq & \mathrm{Y} \varepsilon
\end{aligned}
$$

where $\mathrm{Y}=\frac{2-\mathcal{L}_{g}+\mathcal{K}_{f}+\mathcal{K}_{g}-\mathcal{L}_{f}}{\Delta} \mathrm{Y}_{1,2}$ and $\varepsilon=\max \left\{\varepsilon_{1}, \varepsilon_{2}\right\}$.

\section{Examples}

Consider the $\vartheta$-Hilfer type system

$$
\left\{\begin{array}{cl}
\mathbb{D}_{0^{+}, \frac{1}{3}, \frac{1}{3}}^{\frac{1}{3}} v(\varkappa)=f\left(\varkappa, v(\varkappa), \mathbb{I}_{a^{+}, \vartheta(\varkappa)}^{\rho_{3}} \omega(\varkappa)\right), & \varkappa \in(0,1], \\
\mathbb{D}_{0^{+}, \frac{\varkappa}{3}, \frac{1}{3}}^{\frac{1}{3}} \omega(\varkappa)=g\left(\varkappa, \omega(\varkappa), \mathbb{I}_{a^{+}, \vartheta(\varkappa)}^{\rho_{3}} v(\varkappa)\right), & \varkappa \in(0,1], \\
\left.\mathbb{I}_{0^{+}, \frac{\varkappa}{3}}^{\frac{1}{2}} v(\varkappa)\right|_{\varkappa=0}=1,\left.\mathbb{I}_{0^{+}, \frac{\varkappa}{3}}^{\frac{1}{2}} \omega(\varkappa)\right|_{\varkappa=0}=2,
\end{array}\right.
$$

where $\rho_{1}=\frac{1}{3}, \rho_{2}=\frac{1}{4}, \rho_{3}=\frac{1}{4}, \gamma=\frac{1}{2}, v_{0}=1$, and $\omega_{0}=2$. 
1. In order to illustrate Theorem 5 , we take $\vartheta(\varkappa)=\frac{\varkappa}{3}$ and

$$
\left\{\begin{array}{l}
f\left(\varkappa, v(\varkappa), \mathbb{I}_{a^{+}, \vartheta(\varkappa)}^{\rho_{3}} \omega(\varkappa)\right)=\frac{8}{20}\left(\sin v(\varkappa)+\sin \left(\mathbb{I}_{0^{+}, \frac{1}{3}}^{\frac{1}{4}} \omega(\varkappa)\right)+1\right), \\
g\left(\varkappa, \omega(\varkappa), \mathbb{I}_{a^{+}, \vartheta(\varkappa)}^{\rho_{3}} v(\varkappa)\right)=\frac{1}{30}\left(\cos \varkappa+\omega(\varkappa)+\sin \left(\mathbb{I}_{0^{+}, \frac{1}{3}}^{\frac{1}{4}} v(\varkappa)\right)\right) .
\end{array}\right.
$$

Then we have

$$
\begin{aligned}
& \left|f\left(\varkappa, v(\varkappa), \mathbb{I}_{a^{+}, \vartheta(\varkappa)}^{\rho_{3}} \omega(\varkappa)\right)-f\left(\varkappa, \bar{v}(\varkappa), \mathbb{I}_{a^{+}, \vartheta(\varkappa)}^{\rho_{3}} \bar{\omega}(\varkappa)\right)\right| \\
& \leq \frac{8}{20}|\sin v(\varkappa)-\sin \bar{v}(\varkappa)|+\left|\sin \left(\mathbb{I}_{0^{+}, \frac{\varkappa}{3}}^{\frac{1}{4}} \omega(\varkappa)\right)-\sin \left(\mathbb{I}_{0^{+}, \varkappa}^{\frac{1}{4}} \bar{\omega}(\varkappa)\right)\right| \\
& \leq \frac{8}{20}\left(|v(\varkappa)-\bar{v}(\varkappa)|+\left|\mathbb{I}_{0^{+}, \frac{1}{3}}^{\frac{1}{4}} \omega(\varkappa)-\mathbb{I}_{0^{+}, \frac{\varkappa}{3}}^{\frac{1}{4}} \bar{\omega}(\varkappa)\right|\right)
\end{aligned}
$$

and

$$
\begin{aligned}
& \left|g\left(\varkappa, \omega(\varkappa), \mathbb{I}_{a^{+}, \vartheta(\varkappa)}^{\rho_{3}} v(\varkappa)\right)-g\left(\varkappa, \bar{\omega}(\varkappa), \mathbb{I}_{a^{+}, \vartheta(\varkappa)}^{\rho_{3}} \bar{v}(\varkappa)\right)\right| \\
& \leq \frac{1}{30}\left(|\omega(\varkappa)-\bar{\omega}(\varkappa)|+\left|\sin \left(\mathbb{I}_{0^{+}, \frac{1}{3}}^{\frac{1}{4}} v(\varkappa)\right)-\sin \left(\mathbb{I}_{0^{+}, \frac{1}{3}}^{\frac{1}{4}} \bar{v}(\varkappa)\right)\right|\right) \\
& \leq \frac{1}{30}\left(|\omega(\varkappa)-\bar{\omega}(\varkappa)|+\left|\mathbb{I}_{0^{+}, \frac{1}{3}}^{\frac{1}{4}} v(\varkappa)-\mathbb{I}_{0^{+}, \frac{1}{3}}^{\frac{1}{4}} \bar{v}(\varkappa)\right|\right) .
\end{aligned}
$$

Thus, $\left(\mathrm{Hy}_{1}\right)$ holds with $\kappa_{f}=\bar{\kappa}_{f}=\frac{8}{20}$ and $\kappa_{g}=\bar{\kappa}_{g}=\frac{1}{30}$. From the above data, we obtain $\zeta_{1} \approx 0.33$ and $\zeta_{2} \approx 0.26$. Hence $\max _{\varkappa \in \mathbb{J}}\left\{\zeta_{1}, \zeta_{2}\right\}=\zeta \approx 0.33<1$. Thus, with the assistance of Theorem 5, the system (29) with $f$ and $g$ given by (30) has a unique solution $(v(\varkappa), \omega(\varkappa))$ on $(0,1]$.

2. In order to illustrate Theorem 4 , we take

$$
\left\{\begin{array}{c}
f\left(\varkappa, v(\varkappa), \mathbb{I}_{a^{+}, \vartheta(\varkappa)}^{\rho_{3}} \omega(\varkappa)\right)=\frac{1}{40} v(\varkappa) \sin \omega(\varkappa)+\frac{3}{20} \cos v(\varkappa)\left(\mathbb{I}_{0^{+}, \varkappa}^{\frac{1}{4}} \omega(\varkappa)\right), \\
g\left(\varkappa, \omega(\varkappa), \mathbb{I}_{a^{+}, \vartheta(\varkappa)}^{\rho_{3}} v(\varkappa)\right)=\frac{1}{10+\varkappa} \sin \left(\mathbb{I}_{0^{+}, \frac{1}{3}}^{\frac{1}{4}} v(\varkappa)\right)+\frac{3}{100}\left(e^{-\frac{\varkappa}{2}} \omega(\varkappa)\right) .
\end{array}\right.
$$

It is easy to see that

$$
\begin{gathered}
\left|f\left(\varkappa, v(\varkappa), \mathbb{I}_{a^{+}, \vartheta(\varkappa)}^{\rho_{3}} \omega(\varkappa)\right)\right| \leq \frac{1}{40}|v(\varkappa)|+\frac{3}{20}\left|\mathbb{I}_{0^{+}, \varkappa}^{\frac{1}{4}} \omega(\varkappa)\right|, \\
\left|g\left(\varkappa, \omega(\varkappa), \mathbb{I}_{a^{+}, \vartheta(\varkappa)}^{\rho_{3}} v(\varkappa)\right)\right| \leq \frac{1}{10}\left|\left(\mathbb{I}_{0^{+}, \frac{1}{3}}^{\frac{1}{4}} v(\varkappa)\right)\right|+\frac{3}{100}|\omega(\varkappa)| .
\end{gathered}
$$

So, condition $\left(\mathrm{Hy}_{2}\right)$ is satisfied with $\varphi_{f}=\frac{1}{40}, \bar{\varphi}_{f}=\frac{3}{20}, \varphi_{g}=\frac{1}{10}, \bar{\varphi}_{g}=\frac{3}{100}$. Moreover, $\Lambda=\frac{\sqrt{\pi}}{8 \Gamma\left(\frac{5}{6}\right)}+\frac{9 \sqrt{\pi}}{50 \Gamma\left(\frac{13}{12}\right)}$ and $\aleph_{1} \approx 0.14<1$. Thus, Theorem 4 is applied to system (29) with $f$ and $g$ given by (31).

3. In order to illustrate Theorem 6 , we have from case 1 that $\left(\mathrm{Hy}_{1}\right)$ is satisfied. As has been shown in Theorem 6 , for $\varepsilon_{1}=\frac{1}{2}$ and $\varepsilon_{2}=\frac{1}{4}$, if $(\widetilde{v}, \widetilde{\omega}) \in \mathcal{C}_{\frac{1}{2}, \frac{\varkappa}{3}}([0,1], \mathbb{R}) \times$ $\mathcal{C}_{\frac{1}{2}, \frac{\varkappa}{3}}([0,1], \mathbb{R})$ satisfies

$$
\left\{\begin{array}{l}
\left|\mathbb{D}_{0^{+}, \frac{\varkappa}{3}}^{\frac{1}{3}, \frac{1}{4}} \widetilde{v}(\varkappa)-\frac{8}{20}\left(\sin \widetilde{v}(\varkappa)+\sin \left(\mathbb{I}_{0^{+}, \frac{\varkappa}{3}}^{\frac{1}{4}} \widetilde{\omega}(\varkappa)\right)+1\right)\right| \leq \frac{1}{2}, \quad \varkappa \in(0,1], \\
\left|\mathbb{D}_{0^{+}, \frac{\varkappa}{3}}^{\frac{1}{3}, \frac{1}{4}} \widetilde{\omega}(\varkappa)-\frac{1}{30}\left(\cos \varkappa+\widetilde{\omega}(\varkappa)+\sin \left(\mathbb{I}_{0^{+}, \frac{\varkappa}{3}}^{\frac{1}{4}} \widetilde{v}(\varkappa)\right)\right)\right| \leq \frac{1}{4}, \quad \varkappa \in(0,1],
\end{array}\right.
$$


there exists a unique solution $(v, \omega) \in \mathcal{C}_{\frac{1}{2}, \frac{\varkappa}{3}}([0,1], \mathbb{R}) \times \mathcal{C}_{\frac{1}{2}, \frac{\varkappa}{3}}([0,1], \mathbb{R})$ of the problem (29) with $f$ and $g$ given by (30) such that

$$
\|(\widetilde{v}, \widetilde{\omega})-(v, \omega)\|_{\mathcal{C}_{\frac{1}{2}, \frac{\pi}{3}}} \leq \frac{1}{2} \mathrm{Y} .
$$

where $\mathrm{Y}=\frac{2-\mathcal{L}_{g}+\mathcal{K}_{f}+\mathcal{K}_{g}-\mathcal{L}_{f}}{\Delta} \mathrm{Y}_{1,2}=0.952857>0, \varepsilon=\max \left\{\varepsilon_{1}, \varepsilon_{2}\right\}=\frac{1}{2}, \mathrm{Y}_{1,2}=$ $\max \left\{\mathrm{Y}_{1}, \mathrm{Y}_{2}\right\}=\frac{1}{(3)^{\frac{5}{6}} \Gamma\left(\frac{4}{3}\right)}$

$$
\mathrm{Y}_{1}=\mathrm{Y}_{2}=\frac{1}{(3)^{\frac{5}{6}} \Gamma\left(\frac{4}{3}\right)}>0
$$

and

$$
\begin{aligned}
& \mathcal{L}_{f}=\frac{2 \sqrt{\pi}}{5 \sqrt[3]{3} \Gamma\left(\frac{5}{6}\right)}, \mathcal{K}_{f}=\frac{2 \sqrt{\pi}}{5 \sqrt[12]{3^{7}} \Gamma\left(\frac{13}{12}\right)} \\
& \mathcal{L}_{g}=\frac{\sqrt{\pi}}{30 \sqrt[3]{3} \Gamma\left(\frac{5}{6}\right)}, \quad \mathcal{K}_{g}=\frac{\sqrt{\pi}}{30 \sqrt[12]{3^{7}} \Gamma\left(\frac{13}{12}\right)} .
\end{aligned}
$$

Hence $\Delta=\left(1-\mathcal{L}_{f}\right)\left(1-\mathcal{L}_{g}\right)-\mathcal{K}_{f} \mathcal{K}_{g}=0.88 \neq 0$, which implies that system (29) is $\mathrm{H}-\mathrm{U}$ stable.

\section{Conclusions}

Recently, FDEs have attracted the interest of several researchers with prosperous applications, especially those involving generalized fractional operators. It is important that we investigate the fractional systems with generalized Hilfer derivatives since these derivatives cover many systems in the literature and they contain a kernel with different values that generates many special cases. As an additional contribution in this topic, existence, uniqueness, and U-H stability results of a coupled system for a new class of fractional integrodifferential equations in the generalized Hilfer sense are examined. The analysis of obtained results is based on applying Schauder's and Banach's fixed point theorems, and Arzelà-Ascoli's theorem.

It should be noted that in light of our obtained results, our use of the generalized Hilfer operator covers many systems associated with different values of the function $\vartheta$ and the parameter $\rho_{2}$, as is the case in the Special Cases section.

Author Contributions: Conceptualization, M.S.A., A.M.S. and M.B.J.; formal analysis, M.S.A., A.M.S. and M.B.J.; methodology, M.S.A. All authors have read and agreed to the published version of the manuscript.

Funding: The Deanship of Scientific Research at Qassim University supported this work.

Institutional Review Board Statement: Not applicable.

Informed Consent Statement: Not applicable.

Data Availability Statement: Not applicable.

Acknowledgments: The authors thank the anonymous referees for their careful reading of the manuscript and their insightful comments, which have helped improve the quality of the manuscript. Moreover, the first author would like to thank the Department of Mathematics, College of Science, and the Deanship of Scientific Research at Qassim University for encouraging scientific research and supporting this work.

Conflicts of Interest: The authors declare no conflict of interest. 


\section{References}

1. Diethelm, K. The Analysis of Fractional Differential Equations, Lecture Notes in Mathematics; Springer: New York, NY, USA, 2010.

2. Kilbas, A.A.; Srivastava, H.M.; Trujillo, J.J. Theory and Applications of the Fractional Differential Equations; Elsevier: Amsterdam, The Netherlands, 2006; Volume 204.

3. Miller, K.S.; Ross, B. An Introduction to the Fractional Calculus and Differential Equations; John Wiley: New York, NY, USA, 1993.

4. Samko, S.G.; Kilbas, A.A.; Marichev, O.I. Fractional Integrals and Derivatives; Gordon and Breach Science: Yverdon, Switzerland, 1993.

5. Hilfer, R. Applications of Fractional Calculus in Physics; World Scientific: Singapore, 2000.

6. Abdo, M.S.; Abdeljawad, T.; Shah, K.; Ali, S.M. On nonlinear coupled evolution system with nonlocal subsidiary conditions under fractal-fractional order derivative. Math. Meth. Appl. Sci. 2021, 44, 6581-6600. [CrossRef]

7. Wang, J.; Shah, K.; Ali, A. Existence and Hyers-Ulam stability of fractional nonlinear impulsive switched coupled evolution equations. Math. Meth. Appl. Sci. 2018, 41,1-11. [CrossRef]

8. Ahmad, M.; Zada, A.; Alzabut, J. Hyers-Ulam stability of a coupled system of fractional differential equations of Hilfer-Hadamard type. Demon. Math. 2019, 52, 283-295. [CrossRef]

9. Almalahi, M.A.; Abdo, M.S.; Panchal, S.K. Existence and Ulam-Hyers stability results of a coupled system of $\psi$-Hilfer sequential fractional differential equations. Results Appl. Math. 2021, 10, 100142. [CrossRef]

10. Abbas, S.; Benchohra, M.; Graef, J.R. Coupled systems of Hilfer fractional differential inclusions in Banach spaces. Commun. Pure Appl. Anal. 2018, 17, 2479. [CrossRef]

11. Khan, H.; Khan, A.; Abdeljawad, T.; Alkhazzan, A. Existence results in Banach space for a nonlinear impulsive system. Adv. Differ. Equ. 2019, 2019, 18. [CrossRef]

12. Khan, R.A.; Shah, K. Existence and uniqueness of solutions to fractional order multi-point boundary value problems. Commun. Appl. Anal. 2015, 19, 515-526.

13. Furati, K.F.; Kassim, M.D.; Tatar, N.E. Existence and uniqueness for a problem involving Hilfer fractional derivative. Comput. Math. Appl. 2012, 64, 1616-1626. [CrossRef]

14. Salim, A.; Benchohra, M.; Karapınar, E.; Lazreg, J.E. Existence and Ulam stability for impulsive generalized Hilfer-type fractional differential equations. Adv. Differ. Equ. 2020, 2020, 1-21. [CrossRef]

15. Almeida, R. A Caputo fractional derivative of a function with respect to another function. Commun. Nonlinear Sci. Numer. Simul. 2017, 44, 460-481. [CrossRef]

16. Vanterler, C.S.J.; Capelas, O.E. On the $\psi$-Hilfer fractional derivative. Commun. Nonlinear Sci. Numer. Simul. 2018, 60, 72-91. [CrossRef]

17. Vanterler, C.S.J.; Kucche, K.D.; Capelas, O.E. On the Ulam-Hyers stabilities of the solutions of $\psi$-Hilfer fractional differential equation with abstract Volterra operator. Math. Methods Appl. Sci. 2019, 42, 3021-3032.

18. Luo, D.; Shah, K.; Luo, Z. On the Novel Ulam-Hyers Stability for a Class of Nonlinear $\psi$-Hilfer Fractional Differential Equation with Time-Varying Delays. Mediterranean J. Math. 2019, 16, 1-15. [CrossRef]

19. Wahash, H.A.; Abdo, M.S.; Panchal, K.S. Fractional integrodifferential equations with nonlocal conditions and generalized Hilfer fractional derivative. Ufa Math. J. 2019, 11, 151-171. [CrossRef]

20. Abdo, M.S.; Hanan, A.W.; Panchal, S.K. Ulam-Hyers-Mittag-Leffler stability for a $\psi$-Hilfer problem with fractional order and infinite delay. Results Appl. Math. 2020, 7, 100115. [CrossRef]

21. Abdo, M.S.; Thabet, S.T.; Ahmad, B. The existence and Ulam-Hyers stability results for $\psi$-Hilfer fractional integrodifferential equations. J. Pseudo-Differ. Oper. Appl. 2020, 11, 1757-1780. [CrossRef]

22. Asawasamrit, S.; Kijjathanakorn, A.; Ntouyas, S.K.; Tariboon, J. Nonlocal boundary value problems for Hilfer fractional differential equations. Bull. Korean Math. Soc. 2018, 55, 1639-1657.

23. Ntouyas, S.K.; Vivek, D. Existence and uniqueness results for sequential $\psi$-Hilfer fractional differential equations with multi-point boundary conditions. Acta Math. Univ. Comenianae 2021, 90, 171-185.

24. Sudsutad, W.; Thaiprayoon, C.; Ntouyas, S.K. Existence and stability results for $\psi$-Hilfer fractional integro-differential equation with mixed nonlocal boundary conditions. AIMS Math. 2021, 6, 4119-4141. [CrossRef]

25. Ulam, S.M. Problems in Modern Mathematics; John Wiley and Sons: New York, NY, USA, 1940.

26. Hyers, D.H. On the stability of the linear functional equation. Proc. Natl. Acad. Sci. USA 1941, 27, 222-224. [CrossRef]

27. Obloza, M. Hyers stability of the linear differential equation, Rocznik Nauk-Dydakt. Prac. Mat. 1993, 13, $259-270$.

28. Li, T.; Zada, A. Connections between Hyers-Ulam stability and uniform exponential stability of discrete evolution families of bounded linear operators over Banach spaces. Adv. Differ. Equ. 2016, 2016, 153. [CrossRef]

29. Rus, I.A. Ulam stabilities of ordinary differential equations in a Banach space. Carpathian J. Math. 2010, 26, $103-107$.

30. Kumam, P.; Ali, A.; Shah, K.; Khan, R. Existence results and Hyers-Ulam stability to a class of nonlinear arbitrary order differential equations. J. Nonlinear Sci. Applic. JNSA 2017, 10, 2986-2997. [CrossRef]

31. Wang, J.; Li, X. Ulam-Hyers stability of fractional Langevin equations. Appl. Math. Comput. 2015, 258, 72-83. [CrossRef]

32. Ali, Z.; Zada, A.; Shah, K. On Ulam's Stability for a Coupled Systems of Nonlinear Implicit Fractional Differential Equations. Bull. Malays. Math. Sci. Soc. 2019, 42, 2681-2699. [CrossRef]

33. Kassim, M.D.; Abdeljawad, T.; Shatanawi, W.; Saeed, M.A.; Abdo, M.S. A qualitative study on generalized Caputo fractional integro-differential equations. Adv. Differ. Equ. 2021, 2021, 375. [CrossRef] 
34. Abdo, M.S.; Shah, K.; Panchal, S.K.; Wahash, H.A. Existence and Ulam stability results of a coupled system for terminal value problems involving $\psi$-Hilfer fractional operator. Advances in Difference Equations. Adv. Differ. Equ. 2020, 2020, 316. [CrossRef]

35. Da Vanterler, C.S.J.; Capelas, O.E. On the Ulam-Hyers-Rassias stability for nonlinear fractional differential equations using the $\psi$-Hilfer operator. J. Fixed Point Theory Appl. 2018, 20, 96.

36. Abdo, M.S.; Panchal, S.K. Fractional integro-differential equations involving $\psi$-Hilfer fractional derivative. Adv. Appl. Math. Mech. 2019, 11, 338-359.

37. Andrews, G.E.; Askey, R.; Roy, R. Special Functions; Encyclopedia of Mathematics and its Applications 71; Cambridge University Press: Cambridge, UK, 1999; Volume 71.

38. Granas, A.; Dugundji, J. Fixed Point Theory; Springer: New York, NY, USA, 2003.

39. Smart, D.R. Fixed Point Theorems; Cambridge University Press: Cambridge, UK, 1980.

40. Hadamard, J. Essai sur létude des fonctions donnees par leur developpment de Taylor. J. Mat. Pure Appl. Ser. 1892, 8, 101-186.

41. Qassim, M.D.; Furati, K.M.; Tatar, N.E. On a differential equation involving Hilfer-Hadamard fractional derivative. Abstr. Appl. Anal. 2012, 2012, 391062. [CrossRef]

42. Katugampola, U.N. A new approach to generalized fractional derivatives. Bull. Math. Anal. Appl. 2014, 6, 1-15.

43. Oliveira, D.S.; de Oliveira, E.C. Hilfer-Katugampola fractional derivatives. Comput. Appl. Math. 2018, 37, 3672-3690. [CrossRef]

44. Almeida, R.; Malinowska, A.B.; Odzijewicz, T. Fractional differential equations with dependence on the Caputo-Katugampola derivative. J. Comput. Nonlinear Dyn. 2016, 11, 061017. [CrossRef]

45. Jarad, F.; Abdeljawad, T.; Baleanu, D. Caputo-type modification of the Hadamard fractional derivatives. Adv. Differ. Equ. 2012, 2012, 142. [CrossRef] 